\title{
A scalable data dissemination protocol for both highway and urban vehicular environments
}

\author{
Ramon S Schwartz ${ }^{*}$, Hans Scholten and Paul Havinga
}

\begin{abstract}
Vehicular ad hoc networks (VANETs) enable the timely broadcast dissemination of event-driven messages to interested vehicles. Especially when dealing with broadcast communication, data dissemination protocols must achieve a high degree of scalability due to frequent deviations in the network density. In dense networks, suppression techniques are designed to prevent the so-called broadcast storm problem. In sparse networks, protocols incorporate store-carry-forward mechanisms to take advantage of the mobility of vehicles to store and relay messages until a new opportunity for dissemination emerges. Despite numerous efforts, most related works focus on either highway or urban scenarios, but not both. Highways are mostly addressed with a single directional dissemination. For urban scenarios, protocols mostly concentrate on either using infrastructure or developing methods for selecting vehicles to perform the store-carry-forward task. In both cases, dense networks are dealt with suppression techniques that are not optimal for multi-directional dissemination. To fill this gap, we present an infrastructure-less protocol that combines a generalized time slot scheme based on directional sectors and a store-carry-forward algorithm to support multi-directional data dissemination. By means of simulations, we show that our protocol scales properly in various network densities in both realistic highway and urban scenarios. Most importantly, it outperforms state-of-the-art protocols in terms of delivery ratio, end-to-end delay, and number of transmissions. Compared to these solutions, our protocol presents up to seven times lower number of transmissions in dense highway scenarios.
\end{abstract}

\section{Introduction}

Vehicular ad hoc networks (VANETs) are expected to serve as support to the development of a wide range of applications related to safety, transport efficiency, and even infotainment [1]. Such applications are built upon internal sensor data that is continuously gathered, processed, and disseminated to other vehicles in the neighborhood. Since the acquired data is usually of interest to a number of vehicles in the region, e.g., data about accidents, broadcasting becomes the predominant communication paradigm.

However, several challenges arise when relying on broadcast communication. Broadcasting is particularly unreliable due to the lack of acknowledgments in the carrier sense multiple access with collision avoidance (CSMA/CA) mechanism present in the 802.11p standard. Also, vehicular networks are very dynamic in nature with

*Correspondence: r.s.schwartz@utwente.nl

Pervasive Systems Group, Department of Computer Science, University of Twente, Enschede 7522 NB, The Netherlands large deviations in density depending on the current road traffic. Scalability becomes then a paramount factor to be taken into account when designing data dissemination protocols for VANETs.

In dense networks, a pure flooding scheme results in excessive redundancy, contention, and collision rates [2], which is referred to as the broadcast storm problem. Such a problem is tackled with broadcast suppression techniques. Most of these techniques aim to assign vehicles to different delay values before attempting to rebroadcast that are inversely proportional to their distance to the sender. In this way, only the farthest vehicles would rebroadcast, thereby allowing for quick data dissemination [3]. Vehicles assigned to delay values sufficiently higher to hear a rebroadcast echo can suppress their transmissions. This separation in time is accomplished by means of time slots, where each time slot is equivalent to a message's transmission time.

Conversely, in sparse networks, vehicles may face network disconnections when the transmission range

\section{量 Springer}

(c) 2013 Schwartz et al.; licensee Springer. This is an Open Access article distributed under the terms of the Creative Commons Attribution License (http://creativecommons.org/licenses/by/2.0), which permits unrestricted use, distribution, and reproduction in any medium, provided the original work is properly cited. 
employed cannot reach other vehicles farther in the direction of interest. In such scenarios, protocols should also incorporate a store-carry-forward mechanism to take advantage of the mobility of vehicles to store and relay messages until a new opportunity for dissemination emerges.

Despite numerous efforts, most related works focus on either highway or urban scenarios, but not both. On one hand, highways are most commonly addressed with a single directional dissemination, as the data generated is assumed to only affect vehicles in one road direction, e.g., upon the event of an accident. However, such an assumption is not valid in urban scenarios, where a complex road grid with multiple road directions must be considered when relaying data messages. On the other hand, protocols designed specifically for urban scenarios usually concentrate on methods for selecting vehicles to perform the store-carry-forward task or rely on infrastructure to support the data dissemination. Nevertheless, in both types of scenarios, protocols still rely on suppression techniques that are not optimal for multi-directional dissemination.

In this work, we fill this gap by proposing the infrastructure-less $A$ daptive $M$ ulti-directional data $D$ issemination (AMD) protocol that works seamlessly in both highway and urban scenarios. The key contributions of this work can be summarized as follows:

- A generalized time slot scheme based on directional sectors to support multi-directional data dissemination. In each sector, the density of time slots is precisely controlled based on our method for single directional dissemination presented in [4].

- A store-carry-forward algorithm to support multi-directional data dissemination. To this end, we borrow concepts first introduced in our method for a single directional dissemination presented in [5].

- A comprehensive simulation campaign with a direct comparison against three state-of-the-art protocols, namely, distributed vehicular broadcast (DV-CAST) [6], Simple and Robust Dissemination (SRD) [5], and urban vehicular broadcast (UV-CAST) [7], under both realistic highway and urban scenarios. In particular, we take a real map fragment from the Manhattan area in New York City, NY, USA, including the shape of buildings that are used to model radio obstacles.

The remainder of this paper is organized as follows. First, we review the literature and outline problems with current data dissemination protocols. Next, we describe the AMD protocol in detail. The results of our performance evaluation is then detailed and discussed. Finally, this work is concluded with a discussion and outline for future directions.

\section{Related work}

Various solutions for VANETs have been proposed to cope with message dissemination under different traffic conditions. In dense networks, various broadcast suppression techniques have been proposed to prevent the so-called Broadcast Storm Problem. The ultimate goal is to select only the set with the minimum number of vehicles to rebroadcast and disseminate a message toward the region of interest.

In the context of mobile ad hoc networks (MANETs), several solutions to address this problem were proposed and outlined in $[2,8]$. In [8], the authors present a comprehensive comparison study of various broadcasting techniques in MANETs organized into four categories: (1) simple flooding methods, without any form of suppression; (2) probability-based methods, that rely on network topology information to assign a probability for each rebroadcast; (3) area-based methods, which use distance information to decide which nodes should rebroadcast; and (4) neighbor knowledge methods, which maintain state on the neighborhood via periodic hello messages to decide on the next forwarding node. However, these solutions are mostly concerned with providing means for route discovery with minimum extra network load and, therefore, do not take into account the highly dynamic environment present on roads, neither exploit specific characteristics of vehicular networks such as the predictable mobility pattern of vehicles' movements.

In VANETs, it is generally assumed that each broadcast data message relates to a certain event of a specific geographical region, and, thus, it is targeted mostly to vehicles traveling through that region. With this goal, protocols that rely on positioning information falling into categories 3 and 4 are most suitable. In category 3 , nodes in the location-based scheme [2] rebroadcast whenever the additional coverage is higher than a pre-defined threshold. In category 4, most protocols require nodes to share onehop or two-hop neighborhood information with other nodes [9-11]. This is particularly not suitable in vehicular environments, since such information can quickly become outdated due to the high speed of vehicles. In addition, adding neighborhood information to periodic messages results in high network overhead. As pointed out in [12], decreasing message overhead is crucial for leaving sufficient bandwidth for even-critical messages. In view of these drawbacks, several protocols have been proposed specifically for VANET applications. Such protocols present lightweight solutions in terms of overhead and elaborate on previous solutions in category 3 such as in [2] in order to control, based on distance, the thresholds determining when vehicles should rebroadcast. In the following, we select and describe a few of these efforts. For a complete survey of solutions, we refer the reader to [13]. 
The common approach to reduce broadcast redundancy and end-to-end delay in dense vehicular networks is to give the highest priority to the most distant vehicles toward the message direction. In [3], three ways of assigning this priority are presented: Weighted p-Persistence, Slotted 1-Persistence, and Slotted p-Persistence. In the first scheme, the farthest vehicles rebroadcast with the highest probability. In the second approach, vehicles are assigned to different time slots depending on their distance to the sender, where vehicles with the highest priority are given the shortest delay before rebroadcasting. Finally, the third approach mixes probability and delay by giving vehicles with the highest priority the shortest delay and highest probability to rebroadcast. In delaybased schemes, vehicles assigned to later time slots have time to cancel their transmissions upon the receipt of an echo. This would be an indication that the information has already been disseminated and redundant rebroadcasts can be suppressed. Notably, to achieve the lowest possible end-to-end delay, deterministic approaches such as Slotted 1-Persistence should be preferred over probabilistic methods such as Weighted p-Persistence and Slotted pPersistence. The reason lies in always guaranteeing that the farthest vehicle is chosen, which is not the case with probabilistic-based methods.

Delay-based schemes have been used in several other works with the goal of reducing rebroadcast redundancy, e.g., [14-16]. In [14], the contention-based forwarding scheme (CBF) is presented. Authors focus on a distributed delay-based scheme for mobile ad hoc networks that requires no beaconing information. In [15], the urban multi-hop broadcast (UMB) protocol is designed to cope with broadcast storm, hidden node, and reliability problems of multi-hop broadcast in urban areas. UMB has a special operation mode for scenarios with intersections. Nevertheless, it relies on the same time slot principle for directional data dissemination.

Although efficient in tackling the broadcast storm problem, delay-based schemes still present scalability issues when not employed with optimal parameters. One clear limitation in most schemes proposed is the inability to dynamically choose the optimal value for the number and boundaries of the time slots used. Time slots are usually matched to geographical regions within the transmission range of the sender. However, this can lead to an uneven distribution of vehicles in each time slot. Since transmissions in a single time slot occur nearly simultaneously (see [17]) and cannot be canceled, the level of rebroadcast redundancy and collision is unnecessarily increased. To cope with collisions, the work in [18] introduces a means to control the number of time slots according to the network density. However, authors do not cope with the problem of nearly simultaneous transmissions in a single time slot. To the best of our knowledge, the DOT scheme presented in our previous work [4] pioneered in proposing a precise control of the time slots' density by exploiting the presence of periodic beacons. Such beacons provide one-hop neighborhood information and are expected to be massively present to increase cooperative awareness in safety applications [19]. Authors in [20] had later a similar insight of time slots' density control with the DAZL protocol.

Another problem when relying on time slots schemes arises when the message must be disseminated to multidirections, as shown in Figure 1. In Figure 1a, vehicles follow a typical time slot scheme based on distance. Therefore, the most distance vehicle from the sender, i.e., vehicle $v_{1}$, has the highest priority to rebroadcast in the neighborhood. However, such a naive solution clear prevents the dissemination of the message to both north and south directions, as vehicles $v_{2}, v_{3}$, and $v_{4}$ would cancel their rebroadcasts upon hearing the early transmission from $v_{1}$. The same problem occurs in a highway scenario as shown in Figure 1b, where the rebroadcast performed by $v_{1}$ prevents the dissemination of the message to the other direction where vehicles $v_{2}$ and $v_{3}$ are located. This problem is addressed in [21], however, with no support for disconnected networks.

All suppression schemes still depend on additional measures to cope with sparse disconnected networks when the transmission range does not reach farther vehicles in each possible road direction. The typical approach to cope with disconnected networks is to assign selected vehicles the task of storing, carrying, and forwarding messages when new opportunities emerge. The storecarry-forward paradigm is mostly present in works falling in the area of delay-tolerant networks (DTN) and opportunistic networks. In its simplest form, an epidemic routing is used [22], where flooding is used to disseminate messages throughout the network. In this approach, nodes exchange data as soon as new neighbors are discovered. The spray routing [23] generates only a small number of message copies in order to ensure that the number of transmissions are small and controlled. In the context of pocket switched networks (PSNs), where the nodes are devices carried by people, the BUBBLE algorithm is proposed [24]. It takes into account people's social relationships to select the nodes that can best relay messages. However, these approaches were designed assuming a different mobility model from the one present in VANETs, as they usually consider a combination of the mobility of pedestrians, bicycles, and cars. In VANETs, the mobility of vehicles is constrained to single or multiple roads and by well-defined rules. Therefore, in order to achieve optimal results, more tailored solutions are needed.

A few works apply the store-carry-forward mechanism specifically for VANETs $[5-7,25,26]$. In [6], the DV-CAST 


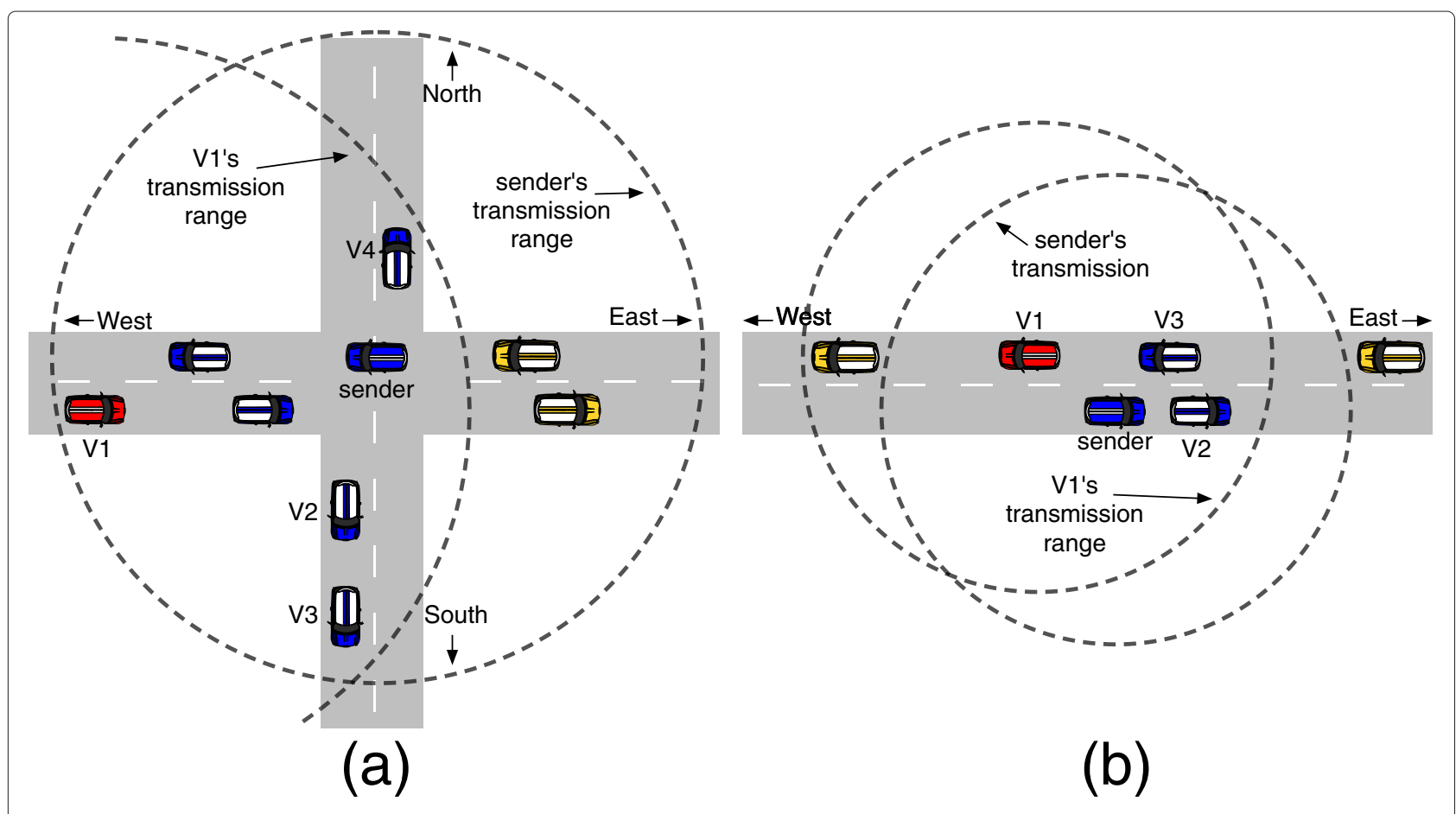

Figure 1 Problem when using a typical time slot scheme for multi-directional dissemination for both urban (a) and highway scenarios (b).

protocol is presented with a combination of a suppression technique and a store-carry-forward approach to cope with both sparse and dense networks in highways. The acknowledged parameterless broadcast in static to highly mobile (ackPBSM) [25] relies on the use of connected dominating sets (CDS) to perform the broadcast of messages. In [26], authors present the enhanced message dissemination based on roadmaps (eMDR), a scheme that mitigates the broadcast storm disconnected networks in real urban scenarios. The UV-CAST is a protocol that specifically addresses urban scenarios with zero infrastructure support [7]. Finally, SRD is a protocol that we previously designed for highway scenarios in [5]. Just as with DV-CAST, SRD combines both a store-carry-forward approach and suppression technique to tackle disconnected and dense networks, respectively. Its suppression technique, Optimized Slotted 1-Persistence, relies on an optimized version of the Slotted 1-Persistence suppression method to prevent nearly simultaneous rebroadcasts in a single time slot in dense networks.

Other related approaches are [27,28]. In [27], authors present the delayed flooding with cumulative neighborhood (DFCN) protocol. DFCN evaluates the benefits of retransmitting a certain message based on whether vehicles in the neighborhood already received the message in previous occasions. While the protocol focuses on reducing transmission redundancy, this reduction comes at the cost of a higher latency, which makes it unsuitable for critical emergency applications. A similar approach is taken in [28], where an example of a tree-based protocol applied to vehicular networks is presented. Although the BODYF protocol aims to achieve a higher efficiency in terms of message exchanged, it still inherits the drawback of typical tree-based approaches of having to perform topology maintenance operations.

Most related works mentioned above address either highway or urban scenarios, or sometimes only the broadcast storm problem in dense networks. In this work, we propose a data dissemination protocol that scales properly from sparse to dense networks and that works seamlessly in both highway and urban scenarios.

\section{Adaptive multi-directional data dissemination}

In this work, we address the limitations of current data dissemination approaches with the Adaptive Multidirectional data Dissemination (AMD) protocol. In contrast to existing approaches, AMD works seamlessly in both highway and urban scenarios. To achieve this goal, we focus on the following aspects:

- Adaptive multi-directional dissemination: to achieve an efficient wide-spreading data dissemination, each data message is simultaneously disseminated to multiple directions that are adaptively adjusted according to the local map of the road provided, for example, by a GPS navigation system. In highway 
scenarios, this usually means disseminating a message to both directions of the road, whereas in urban scenarios, a message is disseminated toward all possible directions in the road grid. For instance, a Manhattan-like grid would have four possible directions in a region comprising an intersection.

- Time slot density control: to cope with dense networks, we propose a time slot suppression scheme, where the final goal is to select only the farthest vehicles in each direction considered for dissemination. This time slot assignment is done by following our solution previously presented in [4], where we exploit positioning information of one-hop neighbors to control with precision the time slots' density. Since the suppression of rebroadcasts is done separately for each possible direction, we guarantee a proper dissemination to all directions and prevent situations where the dissemination is hindered due to an early broadcast suppression, as shown in Figure 1.

- Store-carry-forward: to cope with disconnected sparse networks, vehicles that are furthest away in one of the dissemination directions assume the responsibility of carrying, storing, and rebroadcasting the messages received forward to new vehicles that are encountered.

\section{Concept definitions}

To better understand the protocol, we define the following concepts which are used throughout the remaining sections:

Definition 1 (Directional sector). The directional sectors of a vehicle are defined as the virtual geographical sectors within the vehicle's transmission range to which a data message must be disseminated. Each vehicle automatically adjusts its number of directional sectors according to: (1) the current local road map, e.g., two-directional highway or road intersection with four or more directions; and (2) whether there are vehicles present in each of these possible directional sectors. The second condition serves to prevent unnecessary divisions with empty sectors, e.g., in an intersection where no vehicles are present in one of the two crossing roads. Definition 2 (Directional vehicle cluster). Given a directional sector, a directional vehicle cluster is defined as the group of vehicles with multi-hop connectivity that are positioned farther in the direction of the sector considered.

Definition 3 (Directional cluster tail). Given a directional vehicle cluster, the directional cluster tail is defined as the vehicle within the cluster with no radio connectivity with other vehicles positioned farther in the direction considered. Since the dissemination is multi-directional, a vehicle might be the cluster tail of multiple directional sectors simultaneously.

Figure 2 shows an example of how these concepts are applied. For the sake of simplicity, we limit to show the directional sectors for vehicles $v_{2}$ and $v_{5}$ only. Even though vehicle $v_{2}$ is close to an intersection, it divides its transmission range into only two sectors. This is due to the building that serves as radio obstacle and, consequently, $v_{2}$ can only detect vehicle $v_{3}$ as a neighbor, which resembles a two-direction road as normally occurs in a highway scenario. In contrast, $v_{5}$ has four directional sectors as it is in an intersection point and has neighbors positioned in orthogonal directional sectors. Each directional cluster is highlighted with a surrounding rectangle whereas cluster tails are indicated with a vehicle number, namely, vehicles $v_{1}, v_{2}, v_{3}, v_{4}, v_{5}, v_{6}$, and $v_{7}$. We can observe that vehicle $v_{2}$ is the cluster tail of sector $d_{1}$ in contrast to vehicle $v_{5}$ being the tail of both sectors $d_{2}$ and $d_{3}$.

\section{Requirements and assumptions}

In this work, we assume that no roadside infrastructure is available. Although devices alongside the road could certainly help, we concentrate on the case where only vehicles can generate and disseminate data. To this end, we assume that all vehicles are equipped with radio devices which comply with the de facto standard for vehicular communication IEEE 802.11p. As defined in [29], vehicles shall be able to accommodate an architecture that supports a control channel $(\mathrm{CCH})$ and multiple service channels (SCHs). This can be accomplished via either a single radio with an alternate hoping between $\mathrm{CCH}$ and $\mathrm{SCH}$, or via multiradio capability with one dedicated radio for $\mathrm{CCH}$ and one for all SCHs. In this work, we assume the latter case, with all communication being done in $\mathrm{CCH}$ with a dedicated radio device.

AMD is a dissemination protocol that runs on top of the MAC layer, thereby requiring no modification in IEEE 802.11p standard. We assume that two types of messages can be generated and sent by upper layers, namely, data messages and periodic beacons. Data messages are sent only upon the occurrence of an event, e.g., an accident on the road, and are valid within a geographical region and time period as determined by the application. Throughout the text, we will often simply refer to them as messages. In contrast, periodic beacons are continually transmitted by each vehicle at a certain rate. These beacons are defined to be transmitted in the form of WAVE short messages (WSMs), according to the IEEE 1609 Family of Standards for Wireless Access in Vehicular Environments (WAVE) [30,31]. The IEEE WAVE standard determines that these messages carry information such as the data 


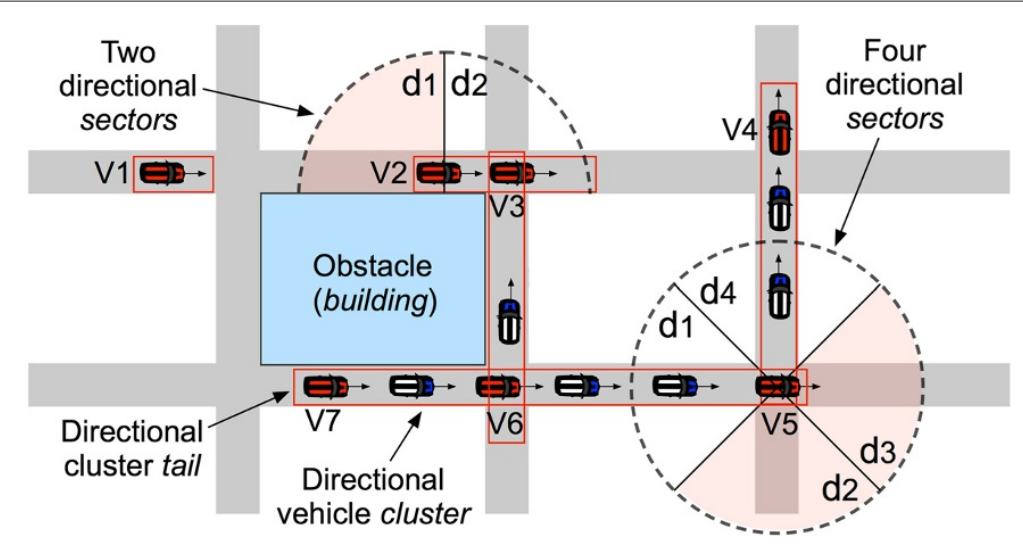

Figure 2 Protocol concepts applied in an urban environment.

rate, channel number, and the transmission power level employed. In addition, contextual information about the vicinity is expected to be included, namely, the vehicle's geographical position, speed, and acceleration [32]. In this work, we assume that each vehicle is equipped with a device capable of obtaining the current vehicle's geographical position, such as a GPS receiver. Finally, we require that vehicles include a message list in their beacons, containing their last $k$ data messages received. This serves to prevent loops in the network, i.e., a continuous rebroadcasting to new vehicles encountered that already received the data message being disseminated.

In order to accurately define the directions of dissemination, we assume that a vehicle is equipped with a device that provides road mapping information, such as a GPS navigation system. In this way, a vehicle can identify the correct number of directions in its local road context, for example, if it is an intersection in an urban setting or a highway. Such mapping information also serves to identify the boundaries of the region that a message is related to, which is assumed to be defined by the application when a message is generated.

\section{Time slot scheme}

To cope with the broadcast storm problem, we adapt the Distributed Optimized Time (DOT) slot scheme that we previously proposed in [4] to the general case of multidirectional data dissemination.

By gathering the information contained in beacons, each vehicle keeps a table of one-hop neighbors $T_{n}$ containing the latest information about the vicinity. Each entry in $T_{n}$ contains the following information: <Vehicle ID, Expiration Time, Vehicle's Geographical Coordinates, Message List $>$. The expiration time field is used to remove vehicles from the table that are no longer in the vicinity. Since there may be failures (e.g., collisions) when sending these beacons, we introduce a time tolerance before removing an entry defined as $t_{t}=2.5\left(\frac{1}{b_{f}}\right)$, where $b_{f}$ is the beaconing rate, e.g., $10 \mathrm{~Hz}$. This accounts for failure in one beaconing period plus possible extra delay. The message list keeps track of the $k$ last messages received by each neighbor.

The time slot scheme works as follows. Let $i$ be the vehicle sender of message $m$, and $D$ be the set of directional sectors to which $m$ must be disseminated. In addition, let $R$ be the set of vehicles receiving $m$ and $R_{d} \in R$ be the sub-set of vehicles receiving $m$ within directional sector $d \in D$. Every vehicle $j \in R$ receiving $m$ for the first time schedules a rebroadcast for $m$ with a time delay $T_{S i j}$. Whenever a vehicle $j \in R_{d}$ receives an echo of $m$ before $T_{S i j}$ expires from another vehicle $k \in R_{d}$ that is farther in the directional sector $d$, it cancels (suppresses) its rebroadcast. Otherwise, the rebroadcast is performed when $T_{S i j}$ expires.

The process of defining $T_{S i j}$ involves two tasks performed by the sender before transmitting $m$. The first task involves estimating which vehicles in the neighborhood will receive $m$, i.e., belong to set $R$. This is achieved by using the power level used to send message $m$, which allows to estimate the distance that $m$ will travel and, thus, which neighbors in $T_{n}$ will be reached by $m$. The second task involves defining the order in terms of priority that each vehicle in $R$ should attempt to rebroadcast $m$. For this purpose, we use the common criteria of assigning a higher priority to the most distant vehicles relatively to the sender. However, in order to give equal importance to each directional sector $d \in D$ considered, the final order of rebroadcasts is defined in a round-robin fashion where the farthest vehicle in directional sector $d_{1}$ transmits first, followed by the farthest vehicle in $d_{2}$, and so forth. The final order is stored in list $\vec{v}$ and included in message $m$. In case different vehicles are equally distant from the sender in a single directional sector, they are then additionally sorted by their vehicle 
ID, where lower ID values are placed in front positions in $\vec{v}$.

Figure 3a,b exemplifies this sorting algorithm for both an urban and highway scenarios. In Figure $3 \mathrm{a}$, the transmission range of the sender is divided into four directional sectors, since there are vehicles positioned in each possible directional sector in the intersection. The final order of transmission is defined as $\vec{v}=<$ $v_{1}, v_{2}, v_{3}, v_{4}, v_{5}, v_{6}, v_{7}, v_{8}>$. Vehicles $v_{9}$ and $v_{10}$ are not included, since they are out of the estimated set $R$. In this way, the farthest vehicles in each directional sector have the highest priority. The same pattern is shown for a highway scenario in Figure 3b. The difference lies in dividing the transmission range into only two-directional sectors.

The decision of centralizing both tasks in the sender contrasts with our original approach in [4], where we presented a distributed sorting algorithm for a single directional dissemination. However, when considering a multi-directional dissemination, the use of a centralized decision is paramount to cope with the hidden terminal problem, as motivated in [21]. Figure 4 shows the same highway scenario previously shown in Figure $3 \mathrm{~b}$ but now immediately later in time after all vehicle have already received the message from the sender. If the farthest vehicles $v_{1}$ and $v_{2}$ were to estimate in a distributed fashion which other neighbors also received the message from the sender, they would clearly not include each other in set $R$, as they are out of range. This would result in both vehicles rebroadcasting simultaneously, thereby leading to a collision in the sender. With no echo correctly received, the sender would in turn assume that its previous broadcast failed and the same message would be broadcast once again. To prevent such collisions, the priority list $\vec{v}$ as estimated by the sender is included in $m$ to guarantee a consistent assignment of priority among all receiving neighbors. This comes at the cost of an extra overhead but only in the data message, thereby not including this information in periodic beacons.

Upon receiving message $m$, each vehicle $j \in R$ finds its own position in the received $\vec{v}$. We denote this position as $S_{i j} \in[0, n-1]$, where $n$ is the total number of elements in $\vec{v}$. Next, each vehicle calculates the waiting time before rebroadcasting as defined by:

$$
T_{S i j}=s t\left(\left\lceil\frac{\left(S_{i j}+1\right)}{t s_{d}}\right\rceil-1\right)+A D_{i j} .
$$

With Equation 1, each vehicle is assigned to a time slot that is proportional to its priority in the neighborhood, where high priorities are translated into early time slots. Vehicles with lower priority can cancel their rebroadcasts as soon as they hear an earlier transmission of the same message scheduled. However, such suppression is only done if the echo was sent by another vehicle residing in the same directional sector, as previously mentioned. With these measures, we guarantee the message dissemination in each possible direction while minimizing the delay and number of transmissions.

The main parameter $t s_{d}$ determines the number of vehicles that are allowed to be assigned simultaneously to a single time slot. In other words, this parameter enables the control of time slots' density. To allow the suppression of rebroadcasts scheduled in later time slots, the slot time $s t$ is defined as the total time taken for the transmission to complete and the message be fully received by others, accounting for medium access delay, transmission delay, and propagation delay.

Assigning different time slots to vehicles clearly helps break the synchronization present in a plain flooding, where all vehicles would rebroadcast nearly simultaneously. However, a similar synchronization on a smaller scale can still occur when multiple vehicles are assigned to a single time slot, thereby leading to possible collisions. This problem was referred to as the Timeslot Boundary Synchronization Problem in [17]. This occurs in our approach when $t s_{d}>1$. To cope with this problem, we introduce an additional delay $\mathrm{AD}_{i j}$ defined as:

$$
\mathrm{AD}_{i j}=d\left(S_{i j} \bmod t s_{d}\right),
$$

where $d$ is a time delay sufficiently long for vehicles assigned to the same time slot to sense if other vehicle has already started its transmission and, at the same time, sufficiently low not to overlap with the beginning of later time slots, i.e., $d \ll s t$. Example of possible values that meet these requirements are the SIFS and DIFS parameters in the MAC 802.11p.

We use Figure $3 \mathrm{~b}$ as reference to explain how our mechanism works when different values of $t s_{d}$ are used. With $t s_{d}=1$, each vehicle in the range of the sender $i$ is assigned to a separate time slot based on its distance to the sender. Thus, vehicle $v_{1}$ is assigned to the earliest time slot $\left\{T_{S i 1}=0\right\} ; v_{2}$ to the second time slot $\left\{T_{S i 2}=s t\right\}$; $v_{3} \rightarrow\left\{T_{S i 3}=2 s t\right\}$, and finally $v_{4} \rightarrow\left\{T_{S i 4}=3 s t\right\}$. In contrast, when $t s_{d}=2$, two vehicles are assigned to each single time slot. However, to prevent nearly simultaneous rebroadcasts among the two vehicles in each time slot, the vehicle with higher $S_{i j}$ in the time slot, i.e., lower priority, waits the additional delay $\mathrm{AD}_{i j}=d$. The final assignment is defined as $v_{1} \rightarrow\left\{T_{S i 1}=0\right\} ; v_{2} \rightarrow\left\{T_{S i 2}=d\right\}$; $\nu_{3} \rightarrow\left\{T_{S i 3}=s t\right\}$; and $v_{4} \rightarrow\left\{T_{S i 4}=(s t+d)\right\}$.

With an accurate estimation of set $R$, optimal results in terms of end-to-end delay are achieved when $t s_{d}$ matches the number of directional sectors: $t s_{d}=4$ in Figure 3a and $t s_{d}=2$ in Figure $3 \mathrm{~b}$. This is expected since the farthest vehicles, i.e., one in each direction, rebroadcast almost immediately. As explained, their transmissions are separated in time only by the additional delay $\mathrm{AD}_{i j}$.

The complete broadcast suppression scheme is shown in Figure 5. Whenever a vehicle receives a data message, 


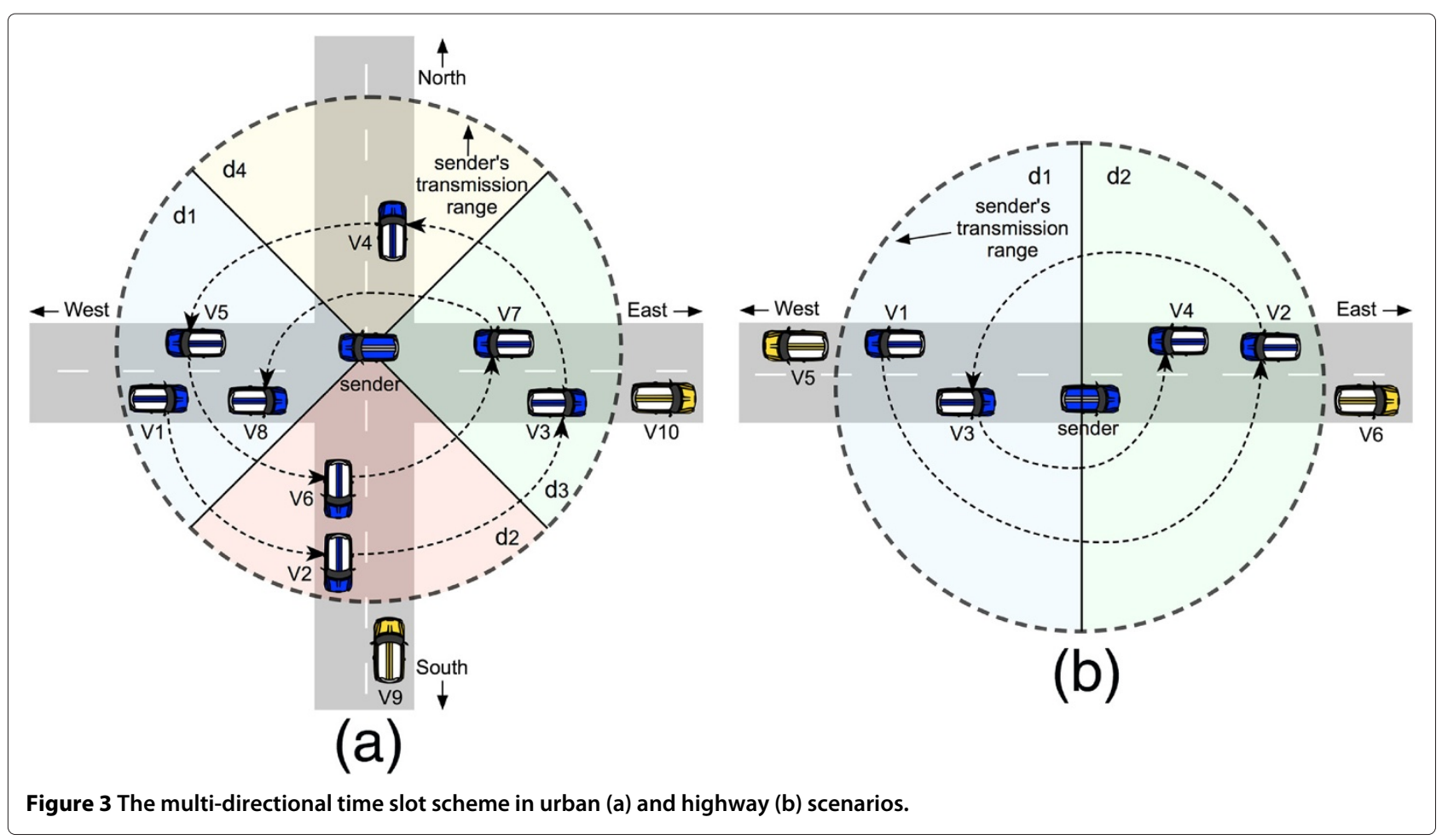

it first checks whether this message comes from a vehicle that is farther in the directional sector defined by the previous sender. The goal is to identify if the vehicle receiving the message is situated in between the current and previous sender, which would indicate that this message has already been disseminated in this geographical region and that a rebroadcast scheduled can be safely canceled (suppressed). In this way, we guarantee that only transmissions scheduled in directional sectors already covered by the message are canceled. This verification is possible, since we include positioning and directional sector information of the previous sender in every data message rebroadcast, as we elaborate in the following sections. If this verification returns false, it means that the receiving vehicle is farther in the directional sector of the previous sender and can schedule a rebroadcast at time $T_{S i j}$ with respect to directional sector of the current sender, if the message has been received for the first time.

\section{Dealing with estimation errors}

As discussed in the previous section, our time slot scheme depends on accurately estimating which vehicles are within the transmission range of the sender, i.e., belong to set $R$. Two main factors can negatively affect this estimation: (1) inaccurate positioning of vehicles in $T_{n}$ due to measurement errors; and (2) inaccurate estimation of the effective transmission range due to path loss affects in wireless communication such as free-space loss, shadowing, and Doppler effect. While the accuracy of a positioning device such as GPS is generally fixed in the order of a few meters, i.e., $5 \mathrm{~m}$ in outdoor environments [33], in wireless communication, the communication range estimation mainly depends on how close the radio propagation model assumed is to reality. Although mapping information is assumed to be potentially available, 3D shapes of buildings are generally unknown $a$ priori by most navigation systems. Therefore, we do not consider obstacles in the calculation of the rebroadcast priority in the neighborhood. However, as highlighted in Figure 2, obstacles will also hinder the reception of beacons sent by vehicles which are directly blocked by them, e.g., vehicles behind buildings. The consequence is that only neighbors previously detected are considered, thereby minimizing estimation inaccuracies when shadowing is present.

On one hand, underestimated transmission range values may lead to vehicles beyond the estimated set $R$ still receiving the message broadcast. Clearly, letting these vehicles rebroadcast would result in an excessive number of transmissions occurring near simultaneously, since they have not been coordinated into different transmission priorities. Instead, we introduce the following policy. If a vehicle $j$ is beyond the range estimated, it is assigned to the last position in list $\vec{v}$. If $\vec{v}$ is empty, $j$ transmits immediately after a random small delay taken from the interval $[0, d]$. This policy may increase the end-to-end delay but it maintains the protocol robust against collisions and contention. On the other hand, overestimated values may 


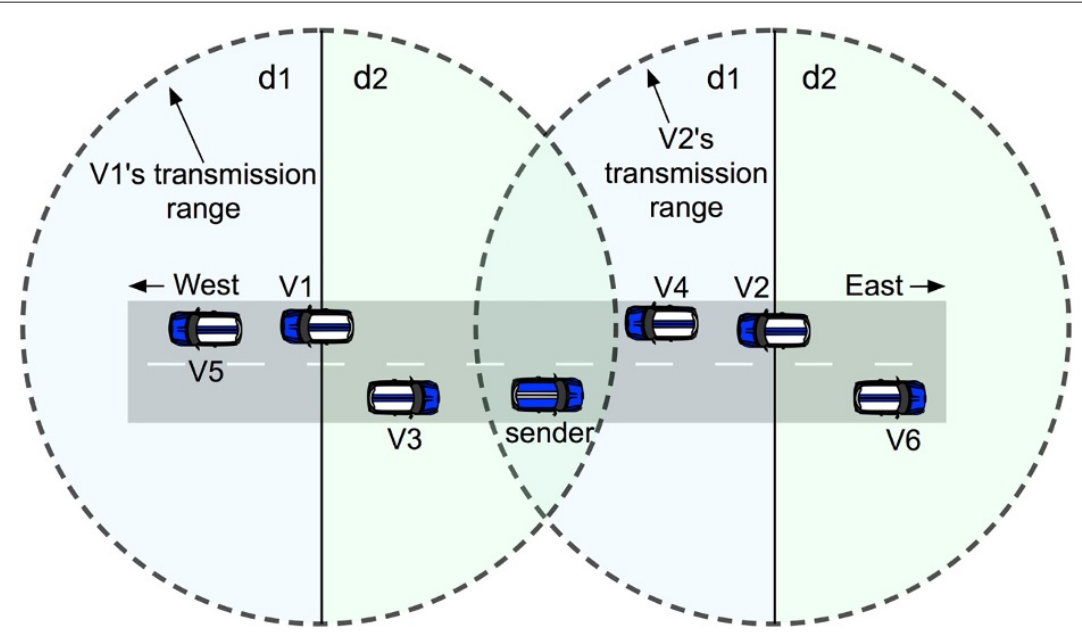

Figure 4 The hidden terminal problem when relying on a distributed sorting algorithm.

result in longer delays, since vehicles unnecessarily wait for the rebroadcast of other vehicles that actually did not receive any message. We tackle overestimated values by being conservative when assuming the maximum distance from the sender that neighbors are still able to receive a message. This can be done by requiring a low outage probability in the propagation model assumed [34].

In [4], we have extensively elaborated on positioning and transmission range estimation and showed by means of simulation that our approach is robust against errors for different time slot density values of $t_{d}$. In this work, our focus is rather on evaluating the new aspects of generalizing our approach to a multi-directional dissemination in both highway and urban scenarios. In this line, one

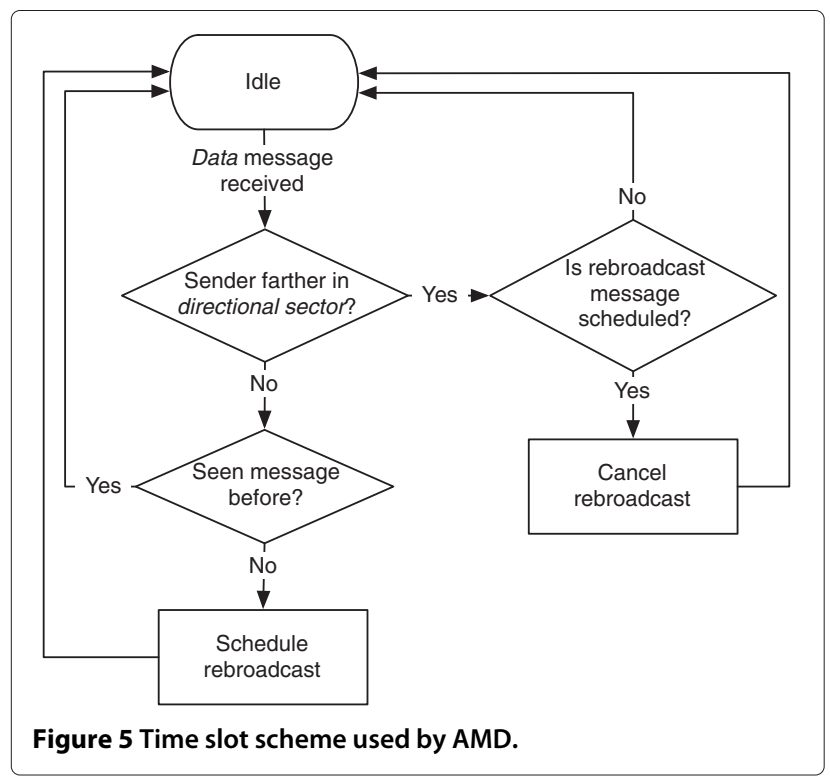

potential source of error lies in the estimation of the number of directional sectors whenever the required mapping information is inaccurate or unavailable. Inaccurate estimates of this value may lead to sub-optimal performance. On the one hand, if an excessive high number of sectors is employed, vehicles are assigned to sectors that do not represent any real road, thereby unnecessarily increasing the number of transmissions. On the other hand, choosing an excessive low number of sectors may lead to the suppression of transmissions of vehicles driving in potential road directions of dissemination, thereby causing higher delays and lower delivery ratio. We elaborate further on these consequences later in the performance evaluation section.

\section{The protocol}

With our proposed time slot scheme, selected vehicles are chosen to rebroadcast whenever new messages are received. In this way, messages are immediately disseminated throughout the network to every possible road direction. However, such a scheme still depends on additional measures to cope with disconnected networks when the transmission range does not reach farther vehicles in the each directional sector.

To cope with radio gaps in the network, we rely on a store-carry-forward approach that is based on our previous single directional dissemination scheme named SRD protocol, presented in [5]. The general idea lies in assigning the responsibility of storing, carrying, and forwarding to vehicles located at the tail of a directional cluster, since these vehicles have the highest probability of meeting later other vehicles farther in the cluster direction. As we exemplified in Figure 2, a vehicle is the tail of a directional cluster if there is no other vehicle farther in that direction. A vehicle can in fact be the tail of 
multiple directional clusters simultaneously, for example, when a vehicle divides its transmission range into four sectors in an intersection as it occurs with $v_{5}$ in Figure 2.

The complete AMD protocol combines both our proposed time slot broadcast suppression and store-carryforward schemes, as shown in Figure 6. Every vehicle updates its local neighborhood information $T_{n}$ with the content received from either a beacon or a data message. When a data message is received, our time slot scheme is executed as defined by the diagram in Figure 5 . On the other side of the diagram, beacons are used to update the tail status of the receiving vehicle for each of its directional sectors. When a vehicle makes the transition from tail to non-tail in one of its directional sectors, it is an indication that there is now connectivity to farther vehicles in that direction and that previously stored messages can be relayed. To prevent unnecessary rebroadcasts, the message list received in the neighbor's beacon is examined and only messages not yet received by the neighbor are rebroadcast.

\section{Defining directional sectors}

Dividing the transmission range into directional sectors is a crucial task done by the sender in order to determine the rebroadcast priority of the receiving neighbors. Such division is achieved by means of the reference vector $\vec{a}$ and the total number of sectors $b$, where $b$ is defined according to

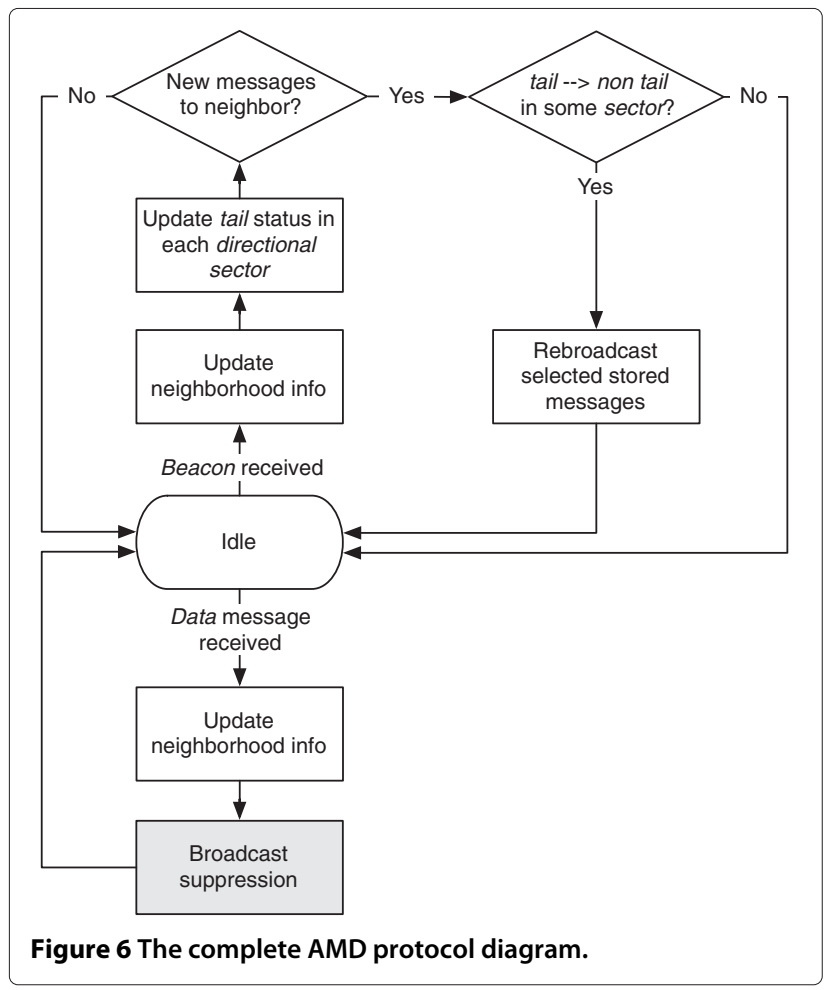

the total number of road directions in the region nearby the sender and whether vehicles in the neighborhood have been detected (via beacons) in each road direction, as motivated in Figure 2.

Figure 7 shows how such division is done. The sender uses its previous and current geographical positions to establish its velocity vector $\vec{v}$. Depending on the number of directional sectors considered $b, \vec{v}$ is rotated in $\beta$ degrees to maximize the road area covered by the sector. In this work, we consider a rotation defined as $\beta=360 / 2 b$, although more appropriate rotation formulas may be considered when more complex road shapes are present. The directional sector that each receiving neighbor belongs is the defined by the angle between the rotated vector $\vec{a}$ and the direction vector $\vec{r}$ with respect to the sender's position. In this example, four directional sectors are considered in the intersection, which yields a rotation of $\beta=45^{\circ}$. Vehicles $v_{1}$ and $v_{2}$ have an angle of $\theta_{1}$ and $\theta_{2}$ between $\vec{a}$ and their vector with respect to the sender $\vec{r}_{1}$ and $\vec{r}_{2}$, respectively. By convention, we define that the index number of directional sectors increases anti-clock wise as in the regular unit trigonometric circle.

\section{Message structure}

Both data messages and beacons have vehicle and message IDs to enable vehicles to distinguish different broadcast messages. An example of vehicle ID is the MAC address, while the message ID can either be a sequence number or a timestamp of the message generation time.

The complete data message structure comprises the following information: <Vehicle ID, Message ID, Vehicle's

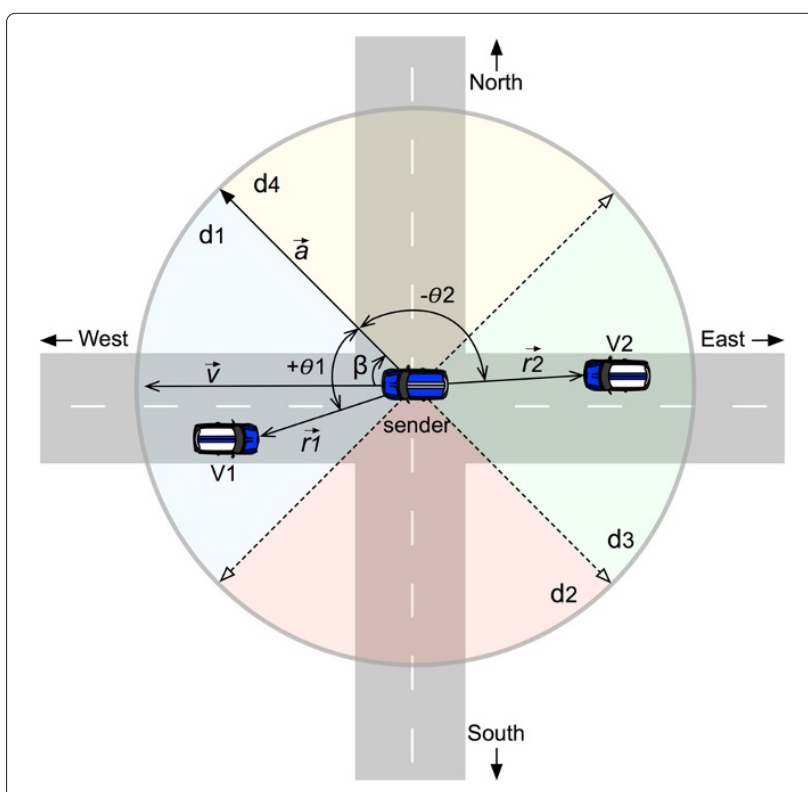

Figure 7 Example of how directional sectors are defined. 
Geographical Coordinates, Time Stamp, Event's Geographical Coordinates, Priority List $\vec{v}$, Previous Direction Reference Vector $\vec{a}_{p}$, Previous Number of Sectors $b_{p}>$. The Time Stamp and Event's Geographical Coordinates fields are used to set the validity for the message in terms of time and distance, respectively. This prevents both the circulation of old messages and that messages travel beyond the boundaries defined by the application. As explained previously, in order for vehicles to suppress their scheduled rebroadcasts correctly, they must know whether an echo of a message comes from a vehicle that is farther in the directional sector defined by the previous sender. For this purpose, every data message also includes the directional sector data of the previous sender, namely, Previous Direction Reference Vector $\vec{a}_{p}$ and Previous Number of Sectors $b_{p}$ fields.

The complete structure of beacons is defined as previously motivated in our requirements section: < Vehicle ID, Message ID, Vehicle's Geographical Coordinates, Message List>.

\section{Performance evaluation}

The performance evaluation of AMD is carried out by means of simulations. Our goal is to study the scalability of AMD under both highway and urban realistic scenarios. We select three state-of-the-art protocols for comparison, namely:

- DV-CAST: it is a protocol designed to cope with both sparse and dense networks in highways [6]. It uses one of the three suppression techniques proposed in [3]. In this work, we set DV-CAST to use the Slotted 1-Persistence suppression technique, which is the mechanism that has shown to achieve best performance in terms of end-to-end delay.

- SRD: it is a protocol that we previously designed for highway scenarios in [5]. Just as with DV-CAST, it combines both a store-carry-forward approach and suppression technique to tackle disconnected and dense networks, respectively. Its suppression technique, Optimized Slotted 1-Persistence, relies on an optimized version of the Slotted 1-Persistence suppression method to prevent nearly simultaneous rebroadcasts in a single time slot in dense networks.

- UV-CAST: it is a protocol that specifically addresses urban scenarios with zero infrastructure support [7]. It combines (1) a suppression technique for dense networks that gives higher priority to vehicles near intersection points; (2) and a gift-wrapping algorithm to select vehicles to store, carry, and forward messages.

We utilize the MiXiM Framework ${ }^{\mathrm{a}}$ and adjust the available implementation of the IEEE $802.11 \mathrm{~b}$ protocol to comply with basic specifications of the 802.11 p version. Table 1 contains a summary of the simulation parameters. In the MAC layer, we set the bit rate to $6 \mathrm{Mbit} / \mathrm{s}$, the Contention Window $(\mathrm{CW})$ to values between 15 and 1,023, the slot time to $13 \mu \mathrm{s}$, the SIFS to $32 \mu \mathrm{s}$, and the DIFS to $58 \mu \mathrm{s}$. In the physical layer, we operate on the $5.88-\mathrm{GHz}$ frequency band, with $10 \mathrm{MHz}$ of bandwidth.

With regard to the transmission power employed, different values may be used according to the application's priority. Efforts put on selecting a proper transmission power value include the decentralized congestion control (DCC) mechanism as defined by the ETSI European standardization [37] that controls the network load by adjusting the transmission power level and transmission rate. However, our goal here is limited to achieving a proper balance between choosing realistic values (i.e., up to $500 \mathrm{~m}$ of range) and achieving scalability in the simulations in terms of the overall processing time. Here,

Table 1 Simulation parameters

\begin{tabular}{|c|c|c|}
\hline \multirow{9}{*}{ Physical layer } & Frequency band & $5.88 \mathrm{GHz}$ \\
\hline & Bandwidth & $10 \mathrm{MHz}$ \\
\hline & Transmission range & $\sim 230 \mathrm{~m}$ \\
\hline & FSPL exponent $\alpha$ & 3.0 \\
\hline & Log-normal $\sigma$ & $6.25 \mathrm{~dB}$ \\
\hline & Obstacle model & Defined in [35] \\
\hline & Receiver sensitivity & $-119.5 \mathrm{dBm}$ \\
\hline & Thermal noise & $-110 \mathrm{dBm}$ \\
\hline & Bit Error Rate (BER) & Based on [36] \\
\hline \multirow{5}{*}{ Link layer } & Bit rate & $6 \mathrm{Mbit} / \mathrm{s}$ \\
\hline & CW & {$[15,1023]$} \\
\hline & Slot time & $13 \mu \mathrm{s}$ \\
\hline & SIFS & $32 \mu \mathrm{s}$ \\
\hline & DIFS & $58 \mu \mathrm{s}$ \\
\hline \multirow{10}{*}{ Suppression mechanisms } & st & $5 \mathrm{~ms}$ \\
\hline & $t s_{d}$ & 1 \\
\hline & $d$ & DIFS \\
\hline & $N S_{\text {std }}$ & 3 \\
\hline & $N S_{\text {opt }}$ & 6 \\
\hline & $D_{\max }$ & $1 \mathrm{~ms}$ \\
\hline & $\tau_{\max }$ & $500 \mathrm{~ms}$ \\
\hline & Beacon frequency & $1 \mathrm{~Hz}$ \\
\hline & Beacon size & $\geq 24$ Bytes \\
\hline & Message list's k & 25 \\
\hline \multirow{3}{*}{ Scenarios } & Data message size & 2,312 bytes \\
\hline & Data message freq. & $0.5 \mathrm{~Hz}$ \\
\hline & \# Runs & 20 \\
\hline
\end{tabular}


we are interested in guaranteeing that multi-hop communication is used in our simulation scenarios in order to properly compare the protocols. Despite leading to higher delay, lower transmission ranges are clearly more suitable to meet this goal. In [4], we evaluate the effects of employing different power levels for different suppression techniques.

Following this reasoning, we set the transmission power to $300 \mathrm{~mW}$ to achieve approximately $230 \mathrm{~m}$ of communication range when assuming the propagation model as described in the following. The bit error rate (BER) model used is the one provided by the Veins project ${ }^{\mathrm{b}}$, which is based on measurements from [38] for the 6-Mbit/s bitrate. We use the Friis Free Space Path Loss (FSPL) propagation model with exponent $\alpha$ equal to 3.0, as it is within the range 2.7 to 5 , estimated for outdoor shadowed urban areas in [39]. We include shadowing effects that are modeled following a log-normal distribution with zero mean and standard deviation $\sigma=6.25 \mathrm{~dB}$, as it is within the range 4 to $12 \mathrm{~dB}$ for outdoor propagation conditions according to [39]. Finally, we use the shadowing obstacle model proposed in [35] to simulate obstacles caused by the presence of buildings in urban scenarios.

For all suppression mechanisms, we set the slot time $s t$ to $5 \mathrm{~ms}$. We define the total number of time slots for Slotted 1-Persistence used by DV-CAST $N S_{\text {std }}$ to 3 and for Optimized Slotted 1-Persistence used by SRD we set $N S_{\text {opt }}$ to 6 (3 slots for each road direction as defined in [5]). The value chosen for Slotted 1-Persistence is based on simulation parameters used in [6]. The maximum additional delay $D_{\max }$ used by Optimized Slotted 1-Persistence is set to $1 \mathrm{~ms}$. For our suppression mechanism, we set the time slot density $t s_{d}$ to 1 and additional delay $d$ to DIFS. For UV-CAST, we set the maximum waiting time parameter $\tau_{\max }$ to $500 \mathrm{~ms}$, as suggested in [7]. Finally, we also map all the intersection points in our urban scenario to allow for a higher priority broadcast by vehicles near intersections, as required by UV-CAST.

For all simulation scenarios, the data message size is 2,312 bytes large, the maximum allowed by the $802.11 \mathrm{p}$ standard. This allows us to evaluate the protocols in the worst-case scenario in terms of medium occupation caused by the transmission of messages. Regarding the message generation frequency, we define that data messages are generated at every 2 s, i.e., message frequency of $0.5 \mathrm{~Hz}$. Although this parameters should be adjusted according to the application requirements, our main concern in this evaluation is to choose a value that gives enough statistical relevance in terms of number of messages generated and at the same time achieve scalability in the simulations, i.e., be able to test a wide range of combinations of scenarios, protocols, and parameters.
The size of beacons can vary from 24 bytes to the maximum message size depending on the message list included. We consider that each new entry in the message list is 12 bytes large, thereby leading to final beacon size of $s(w)=12 w+24$ bytes, where $w$ is the number of entries in the list. Since we limit the total number of entries to $k=25$, the maximum size that each beacon can have in our simulations is limited to 324 bytes. Such limit is chosen based on the proper balance achieved between the number of unnecessary transmissions avoided due to loops in the network and beacon size in the scenarios considered in our simulations. However, further analysis is required to determine the most appropriate value for a wider variety of scenarios.

Beacons are sent at the frequency of $1 \mathrm{~Hz}$. This is usually the highest frequency expected to be used for the transmission of beacons [32], which gives the worst-case scenario in terms of freshness of the one-hop neighborhood information. Furthermore, varying the beaconing rate in our experiments has not led to significant changes in our simulation results, except for more message collisions.

We consider one highway scenario and one urban scenario. The highway consists of a 1-km straight road with two lanes in each road direction. Each message is generated by one fixed vehicle positioned in one end of the road and gathered by another fixed vehicle in the other end of road. For this scenario, in total 20 runs of $100 \mathrm{~s}$ are executed. As urban scenario, we select a map fragment from Manhattan, New York City, USA. This segment has an area of $1.5 \times 2 \mathrm{~km}^{2}$ and was retrieved with OpenStreetMaps ${ }^{\mathrm{c}}$. Messages are generated by one fixed vehicle in the center of the map and gathered by one of the four fixed vehicles that are positioned in each corner of the map. Figure 8 shows the complete map fragment considered, where buildings represented by dark rectangles serve as radio obstacles. Simulations for this urban scenario consist of 20 runs of $300 \mathrm{~s}$.

Both scenarios were created with SUMO [40]. Therefore, they includes realistic mobility patterns such as vehicle overtaking, lane changing, and relies on the wellknown car-following mobility model. Vehicles' speeds vary according to the density considered by following the Krauß mobility model, i.e., the higher the density is, the slower vehicles move.

Our evaluation considers the following metrics:

- Delivery ratio: the percentage of data messages generated that fully propagate the scenario considered until they are received by one of the fixed vehicle responsible to gather data messages. Ideally, dissemination protocols must achieve a delivery ratio percentage close to $100 \%$ in dense networks.

- Delay: the total time taken for a data message generated to fully propagate the scenario considered 


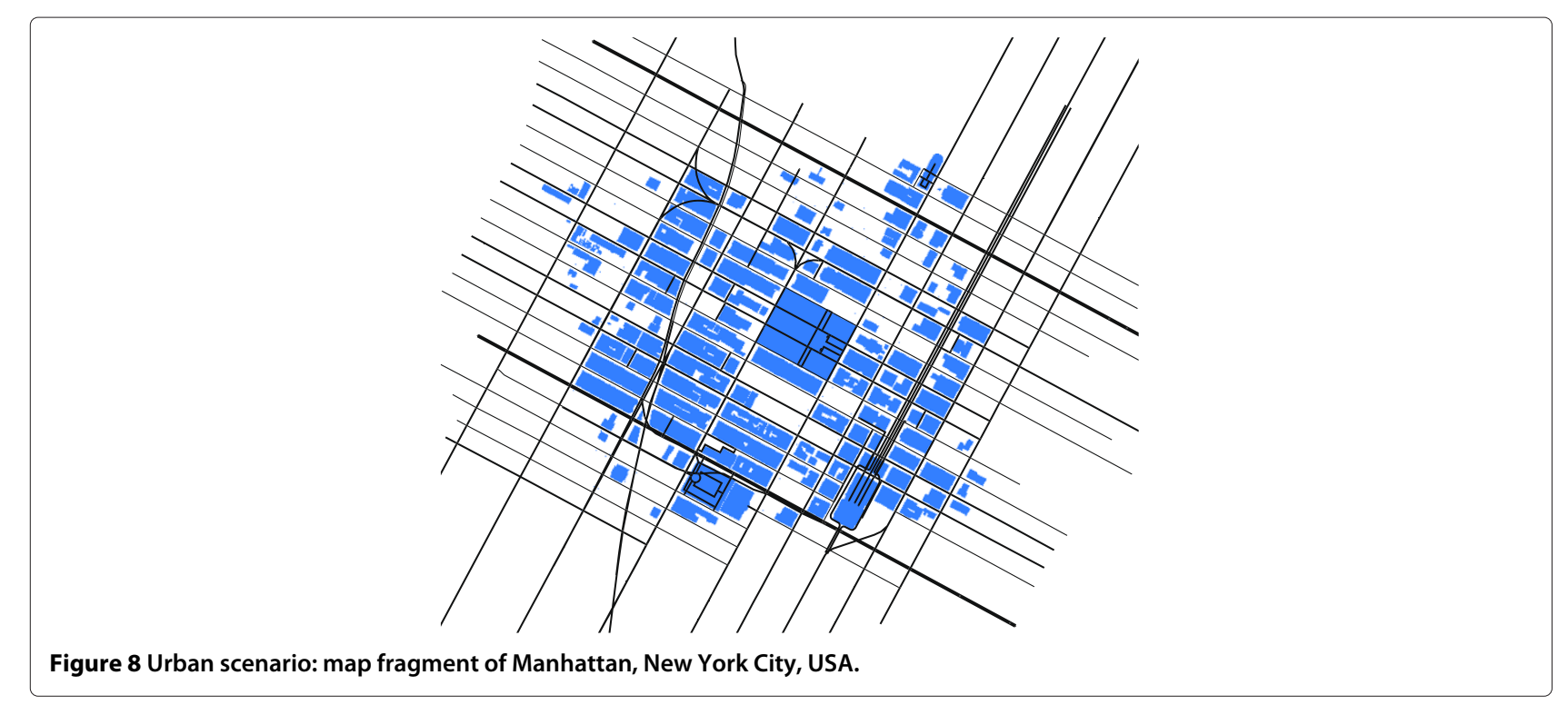

until it is received by one of the vehicles responsible to gather data messages. This is particularly important for critical safety messages that must be disseminated as quickly as possible. We additionally compare the performance of each protocol with a theoretical optimum which serves as lower bound. This value is simply calculated as the minimum number of hops that a message must travel times the transmission delay, given the transmission range employed. We limit this estimation of the theoretical optimum to our highway scenario, since each message has a clear straight trajectory to travel, which leads to a predictable optimum end-to-end delay. The same does not occur for urban scenarios, due to its complexity in terms of multiple possible trajectories, mobility of vehicles, and radio obstacles.

- Total number of transmissions: the total number of transmissions performed on average by an arbitrary vehicle. We consider only data messages in these results, thereby excluding transmissions of beacons. This value is normalized by the total number of vehicles in each scenario. In order to be scalable, protocols must keep a low number of transmissions during a message's dissemination.

\section{Number of directional sectors}

When not properly chosen, the total number of directional sectors can negatively affect the performance of the AMD protocol. As previously explained, AMD uses mapping information provided by a GPS navigation system to adaptively adjust the number of directional sectors according to the number of road directions and the presence of vehicles in the local region. In our simulations, each vehicle is pre-loaded with a simplified version of the scenario map. Such map contains the geographical positions corresponding to the center of each road intersection. Since the scenarios considered contain either straight roads (highway) or follow a Manhattan grid shape (urban scenarios), we define that the number of sectors can be either two or four. More specifically, the number of sectors is four whenever (1) the vehicle about to broadcast is within a radius of $15 \mathrm{~m}$ from the center point of the nearest intersection and (2) at least one neighboring vehicle has been previously detected via the reception of beacons in one of the orthogonal road directions relatively to the velocity vector of the sender. Otherwise, two-directional sectors are employed.

In the following, we analyze the effects of varying the number of directional sectors in both highway and urban scenarios when compared to the adaptive algorithm used by AMD. Figure 9 shows the results for varying the total number of sectors from 2 to 8 . Each number is fixed during the whole simulation run regardless of the number of road directions in the map. In Figure 9a,b, we can observe that choosing a number higher than two for the highway scenario has a negative impact in the delivery ratio and delay. The same occurs for the urban scenario when fixing a number of sectors lower than four. Both results are explained by the fact that choosing two sectors for highways and four or more for urban scenarios provides a better matching to the actual road mapping. On the one hand, an excessive number of sectors leads to too many vehicles being assigned to a different sector. Since the transmission of vehicles can only be suppressed by other vehicles in the same sector, this results in a high number of transmissions and possible collisions in the network (Figure 9c). On the other hand, an excessive low number of sectors leads to an inefficient division of 


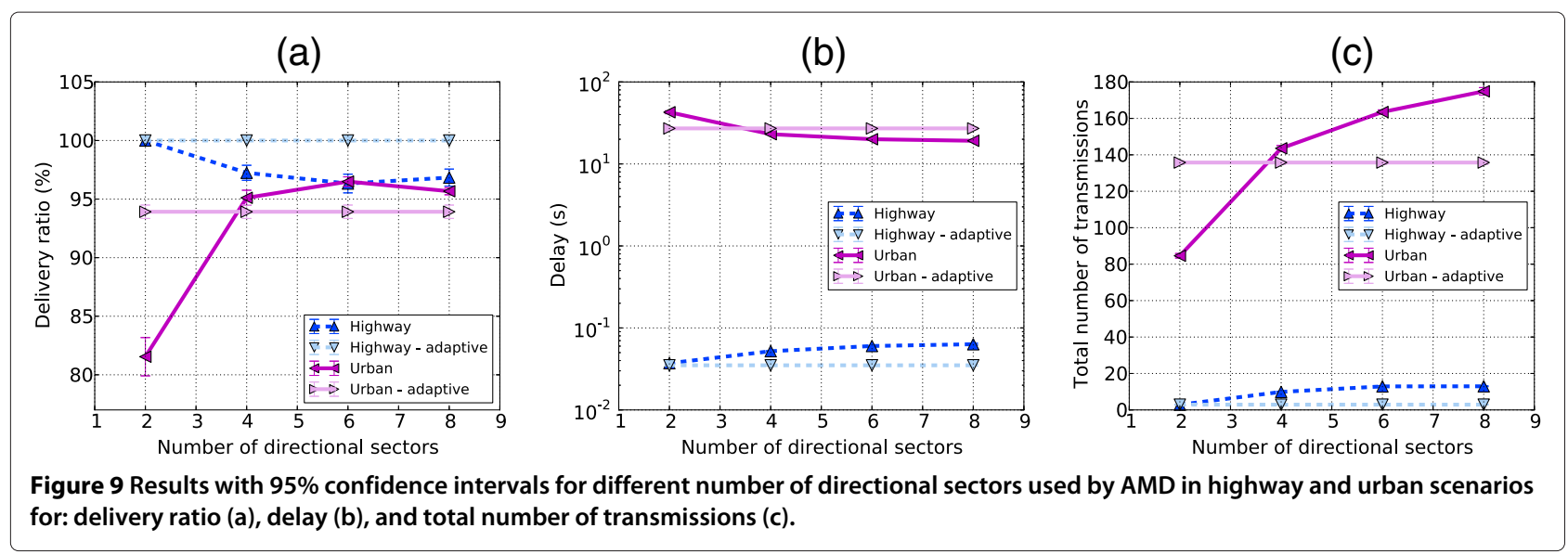

sectors, thereby causing higher delays and lower delivery ratio.

Overall, using mapping information to adaptively choosing the number of directional sectors provides a performance near or equal the best result achieved when fixing the number of sectors beforehand for the whole simulation.

\section{Network density}

In Figures 10 and 11, we show the results for each protocol when varying the network density. Varying the network density evaluates the protocols in terms of scalability, which is crucial in vehicular networks due to its dynamic nature. We additionally show the results for the suppression techniques used by each protocol separately in order to isolate the gains in performance when employing store-carry-forward mechanisms in very low densities.

Figure 10 shows the results for highway scenarios when varying the network density from 1 to $100 \mathrm{~km} / \mathrm{h} / \mathrm{lane}$. We compare AMD with two other protocols designed specifically for highway scenarios, namely, DV-CAST and SRD. As shown in Figure 10a, AMD achieves near
$100 \%$ in delivery ratio for densities higher than 15 vehicles $/ \mathrm{km} /$ lane. In contrast, DV-CAST and SRD present lower delivery ratio, especially in high densities. These protocols lack a means to control the time slots' density, thereby leading to extra rebroadcast redundancy and collisions when many vehicles are assigned to a single time slot. For lower densities, the delivery ratio is lower for all protocols because at the moment that a message is generated, there are cases when no vehicle is in neighborhood to received and disseminate the message to other vehicles in the road. Nevertheless, both AMD and SRD protocols present an improvement of near $45 \%$ in very low densities, namely, density of 5 vehicles $/ \mathrm{km} / \mathrm{lane}$, compared to their suppression techniques alone.

The end-to-end delay tends to increase with density, especially for protocols that rely on a fixed number of time slots such as DV-CAST and SRD, as shown in Figure 10b. The reason lies in the higher contention delay generated when more vehicles attempt to rebroadcast in a single time slot. This can be verified in Figure 10c, where the total number of transmissions is shown to be significantly

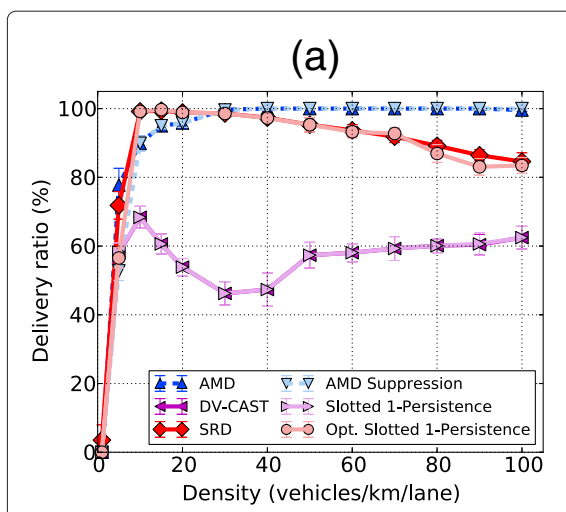

\section{(b)}

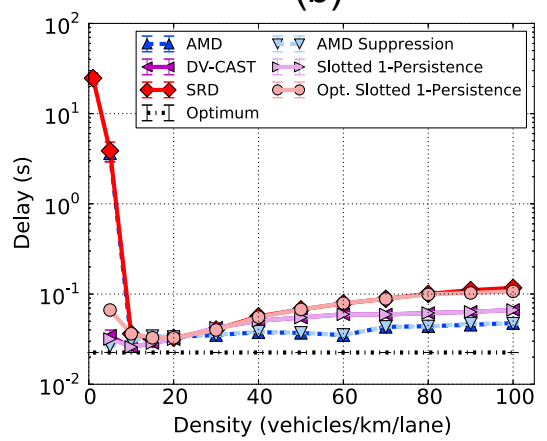

(c)

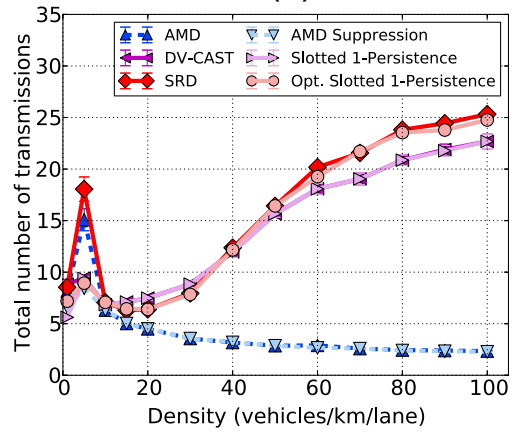

Figure 10 Results with 95\% confidence intervals for increasing network densities in highway scenarios for: delivery ratio (a), delay (b), and total number of transmissions (c). 

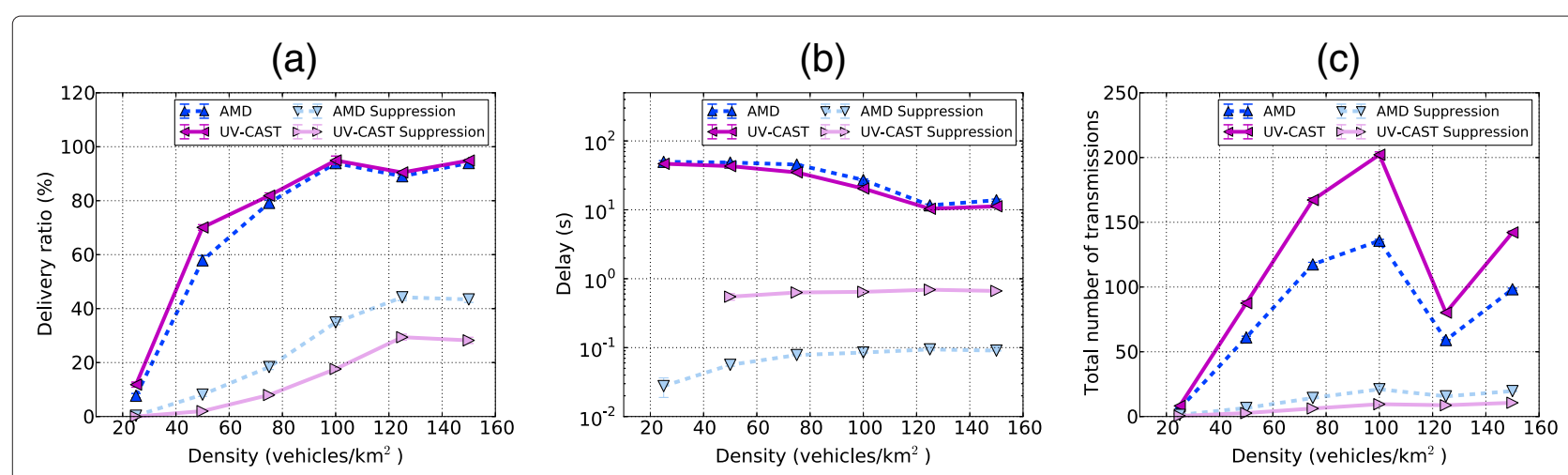

Figure 11 Results with $95 \%$ confidence intervals for increasing network densities in urban scenarios for: delivery ratio (a), delay (b), and total number of transmissions (c).

higher for DV-CAST and SRD. In contrast, in proportion with the total number vehicles in each density, the number of transmissions tends to decrease with AMD thanks to its control of the time slots' density.

The results for our urban scenario when varying the network density from 25 to 150 vehicles $/ \mathrm{km}^{2}$ are shown in Figure 11. AMD is compared against UV-CAST; a protocol designed especially for urban environments. Both protocols achieve similar performance in terms of delivery ratio and end-to-end delay, as shown in Figure 11a,b. This is explained by the fact that when protocols have to resort to using their store-carry-forward mechanisms, their performance in terms of delay and delivery ratio becomes dependent on the movement of vehicles, which is equal for both protocols. However, when verifying the performance of the suppression techniques used by each protocol alone, AMD's suppression clear outperforms the suppression used by UV-CAST in both metrics. This shows that AMD is able to quickly disseminate messages whenever there exist end-to-end connectivity to one of the fixed vehicles responsible for gathering data messages. We can observe that the suppression techniques alone present a lower delivery ratio when compared with their complete protocols. This behavior is particularly expected in urban scenarios where radio obstacles make disconnections predominant, thereby increasing the dependency on store-carry-forward strategies.

In terms of number of transmissions, AMD introduces a lower overhead in the network compared to UV-CAST, as shown in Figure 11c. The reason lies in the ability of AMD to correctly select vehicles to perform the task of carrying and forwarding messages as well as in the ability of its suppression technique to separate vehicles in independent directional sectors, which allows vehicles to properly rebroadcast and suppress transmissions. We can also observe the trend of an increasing number of transmissions from densities 25 to 100 vehicles $/ \mathrm{km}^{2}$. After this point, the network becomes mostly connected and fewer transmissions are needed due to the more frequent use of each suppression technique.

In general, AMD scales more efficiently with increasing network densities when compared with protocols especially designed for either highway or urban scenarios. Compared to these solutions, AMD presents up to seven times lower number of transmissions in dense highway scenarios.

\section{Time slot parameter}

In this section, we analyze the performance of protocols when varying their main parameters, namely, the total number of time slots (used by DV-CAST and SRD), $\tau_{\max }$ (used by UV-CAST) and the time slot density $t s_{d}$ (used by AMD). In particular, SRD uses doubled number of time slots to distribute the number of time slots equally among the two road directions, as detailed in [5]. Contrary to the other protocols, UV-CAST does not define a fixed number of time slots but rather a maximum delay $\tau_{\max }$. In this case, we define that each value in our plot assumes a value of $\tau_{\max }=0.0625 i$, where $i$ falls in the interval from 1 to 8 that is used in the evaluation of each protocol's time slot parameter.

Figure 12 shows the results when varying the time slot parameter of each protocol for highway scenarios. With regard to the delivery ratio, both SRD and DV-CAST achieve higher delivery ratio when increasing the total number of time slots, as shown in Figure 12a. With more time slots, a lower number of vehicles is assigned to a single time slot. Therefore, a lower level of rebroadcast redundancy is expected and messages can travel with less interference throughout the road length. The opposite effect occurs when the time slot density is increased in AMD. Higher values for the number of time slots means more vehicles within a single time slot, which leads to a decrease in delivery ratio from $t s_{d}=4$ in this scenario.

Similarly to what occurs when varying the network density, the end-to-end delay tends to increase when more 


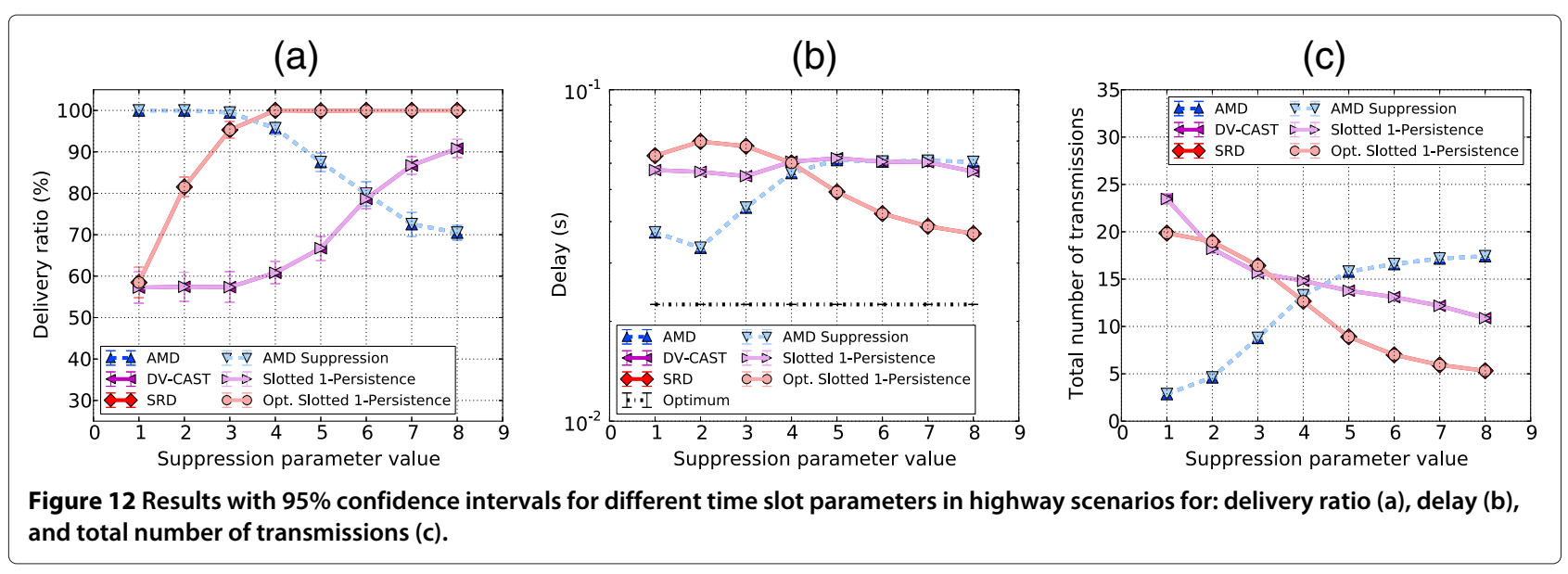

vehicles attempt to transmit nearly simultaneously in a single time slot (Figure 12b). This occurs when decreasing the number of time slots (SRD and DV-CAST) or increasing $t s_{d}$ (AMD). Such an increase in the number of transmissions can be verified in Figure 12c. One interesting remark is that AMD achieves its lowest delay when $t s_{d}=2$, since this allows one transmission in each road direction to occur simultaneously.

With regard to our urban scenario (Figure 13), varying the time slot parameter for both AMD and UV-CAST shows to have little impact when considering their complete protocol with a store-carry-forward mechanism. Their performance is again dependent on the movement of vehicles, which is equal for both protocols.

When looking at each protocol's suppression technique, however, increasing $t s_{d}$ in AMD results in more vehicles being assigned to a single time slot and, thus, in a lower delivery ratio (Figure 13a). Differently from the results for highway scenarios, delay values are lower with higher $t s_{d}$ (Figure 13b), which is a result of the better matching of the number of simultaneous transmission allowed with the multiple road directions present in more complex urban scenarios. In contrast, increasing the $\tau_{\max }$ parameter in UV-CAST implicitly works as increasing the number of time slots used, since it effectively helps spreading the transmissions of vehicles in time. However, because the suppression technique used by UV-CAST is not designed for multi-directional dissemination, such an increase in $\tau_{\max }$ has little impact on the metrics evaluated, apart from the obvious increase in delay.

Overall, all protocols perform best when fewer vehicles attempt to transmit nearly simultaneously. AMD, in particular, presents best performance in terms of delay when the number of simultaneous transmission allowed $t s_{d}$ equals the number of road directions, however, at the cost of a lower delivery ratio in urban scenarios.

\section{Message overhead}

All protocols considered in this evaluation require that a certain overhead is added into beacons or data messages in order to guarantee their proper functioning. Such overhead is generally translated into a fixed number of bytes which correspond to extra fields appended to either

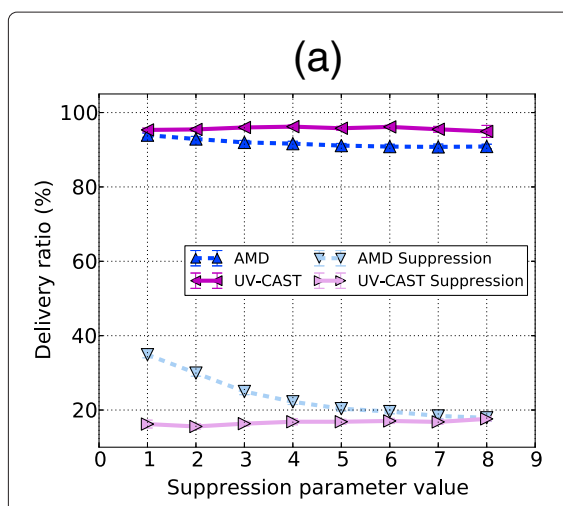

(b)

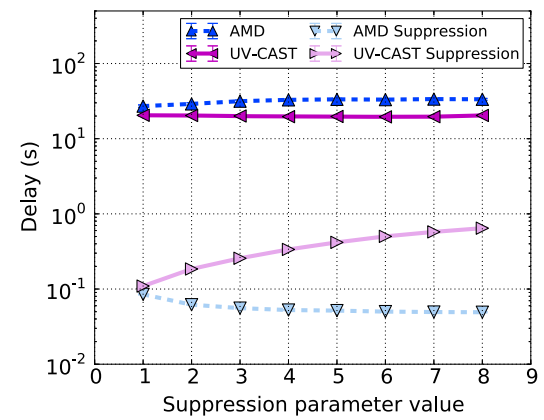

(c)

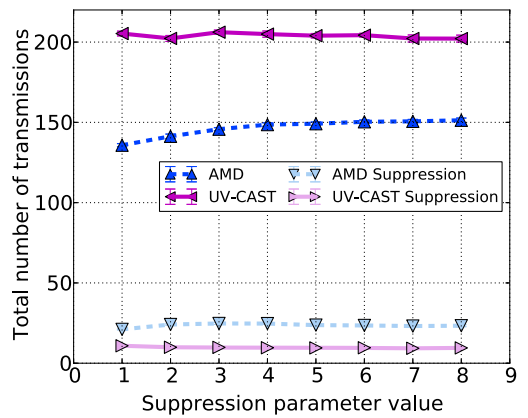

Figure 13 Results with $95 \%$ confidence intervals for different time slot parameters in urban scenarios for: delivery ratio (a), delay (b), and total number of transmissions (c). 
beacon or data messages. However, both AMD and UVCAST resort to appending a message list with variable length to beacons in order to prevent that repeated messages are unnecessarily disseminated in the network. In particular, AMD also includes a small variable list in data messages to guarantee that the order of rebroadcast in the neighborhood is achieved. Therefore, in this last section, we measure the message overhead required by these two protocols when increasing network densities are considered.

Figure 14 shows the message overhead in number of bytes for both protocols in our urban scenario. As explained previously, the size of beacons can vary from 24 bytes to a final beacon size of $s(w)=12 w+24$ bytes, where $w$ is the number of entries in the list. Since we limit the total number of entries to $k=25$, the maximum size that each beacon can have in our simulations is limited to 324 bytes. In Figure 14a, we can observe that this upper bound value is reached when the network density is around 50 vehicles $/ \mathrm{km}^{2}$. Although setting an unbounded value for the number of entries $k$ is obviously unadvisable, we additionally evaluate in this section the total overhead when the maximum list size possible is allowed for each network density. As shown in the same figure, the maximum overhead reached for each density follows a similar pattern as the number of transmissions (Figure 11c). In particular, UV-CAST presents a slightly higher overhead compared with AMD, reaching a maximum of 950 bytes.

With regard to data messages, we compare the extra variable overhead required by AMD with the fixed number of bytes used by UV-CAST. As shown in Figure 14b, such overhead is much lower compared to the message list included in beacons, since it depends only the number of neighbors participating in the rebroadcast operation defined by AMD's suppression technique.

Overall, both AMD and UV-CAST protocols require additional message overhead of variable length to guarantee a proper functioning and to prevent unnecessary transmissions due to potential dissemination loops in the network. Especially for emergency applications, we expect that the message list size introduced in beacons be much lower than what has been considered here, since a single message might be repeated over time by the source vehicle, thereby reducing the number of entries of unique messages in the message list.

\section{Conclusion}

We have presented a data dissemination protocol that works seamlessly in both highway and urban scenar-

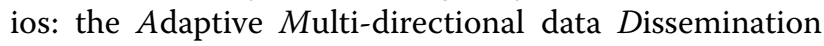
(AMD) protocol. AMD combines a generalized time slot scheme based on directional sectors and a storecarry-forward algorithm to support multi-directional data dissemination.

By means of simulation, we showed that AMD scales properly in various network densities in both highway and urban scenarios. We considered in our simulation scenarios realistic features such as a real map fragment of the Manhattan area in New York City with buildings serving as radio obstacles. Compared with protocols especially designed for either highway or urban scenarios, namely, DV-CAST (highway), SRD (highway), and UV-CAST (urban), AMD obtained higher delivery ratio, lower end-to-end delay, and lower number of transmissions. In particular, AMD presented up to seven (a)

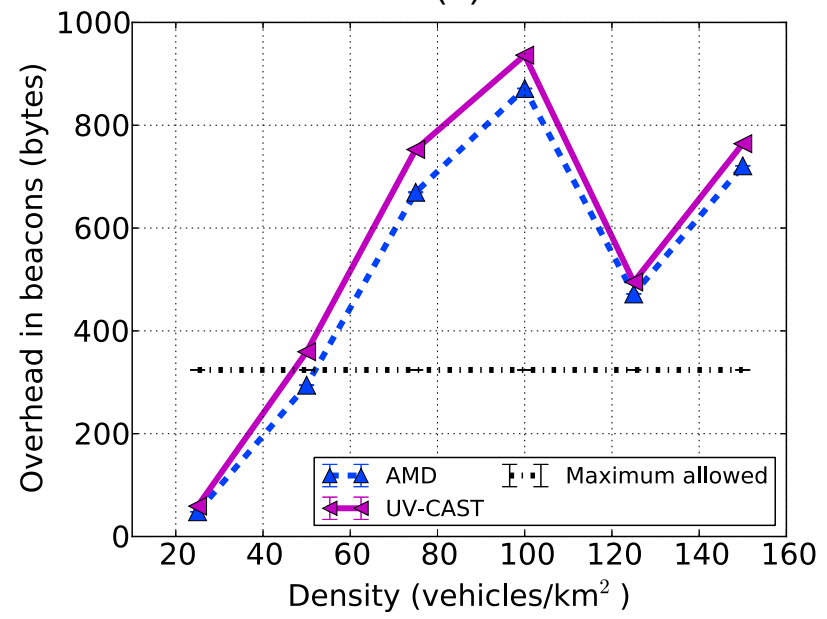

(b)

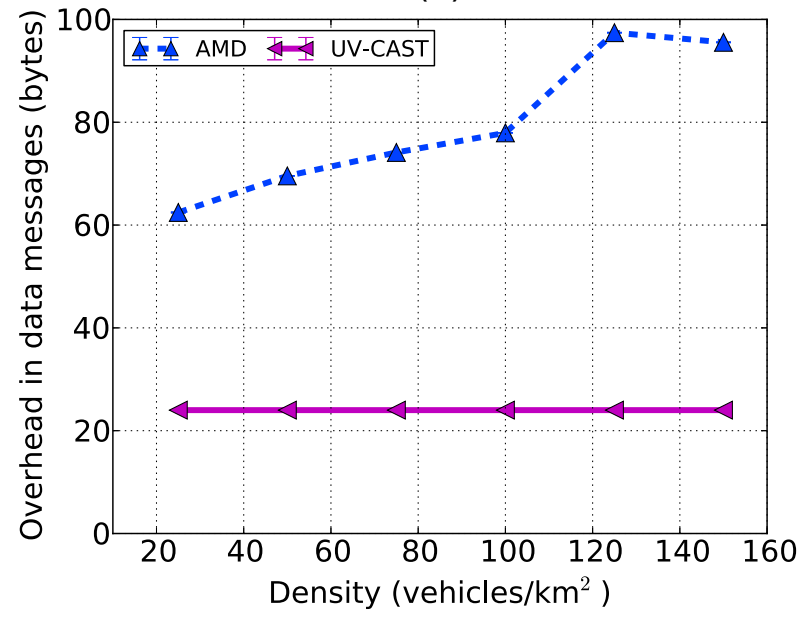

Figure 14 Results with $95 \%$ confidence intervals for the message overhead introduced by AMD and UV-CAST for: beacons (a) and data messages (b). 
times lower number of transmissions in dense highway scenarios.

In this work, we have considered an adaptive algorithm for defining the number of directional sectors for dissemination that is based on the road map and the presence of neighbors in each road direction. However, in our simulations, the focus has been mainly on applying this method on typical straight (highway) or Manhattan grid scenarios (urban). Therefore, one direction for future work is to consider more complex scenarios, e.g., roundabouts with multiple exits or multi-layered highway junctions. In such scenarios, the angle allocated for each direction could be customized and, therefore, flexible to individual attributes of each road direction, for example, by adjusting the angle according to the road width.

In addition, we will aim to consider the support of infrastructure to further improve the end-to-end delay in sparse urban scenarios as well as additional mechanisms to limit the overhead inserted in beacons and data messages.

\section{Endnotes}

a See http://mixim.sourceforge.net

b See http://veins.car2x.org/

c See www.openstreetmap.org

\section{Competing interests}

The authors declare that they have no competing interests.

Received: 19 March 2013 Accepted: 14 October 2013

Published: 4 November 2013

\section{References}

1. G Karagiannis, O Altintas, E Ekici, G Heijenk, B Jarupan, K Lin, T Weil, Vehicular networking: a survey and tutorial on requirements, architectures, challenges, standards and solutions. IEEE Commun. Surv. Tutorials 13(4), 584-616 (2011)

2. SY Ni, YC Tseng, YS Chen, JP Sheu, in Proceedings of the 5th annual ACM/IEEE international conference on Mobile computing and networking (MobiCom). The broadcast storm problem in a mobile ad hoc network (ACM New York, 1999), pp. 151-162

3. N Wisitpongphan, OK Tonguz, JS Parikh, P Mudalige, F Bai, V Sadekar, Broadcast storm mitigation techniques in vehicular ad hoc networks. IEEE Wireless Commun. 14(6), 84-94 (2007)

4. RS Schwartz, K Das, H Scholten, P Havinga, in Proceedings of the ninth ACM international workshop on Vehicular inter-networking, systems, and applications (VANET). Exploiting beacons for scalable broadcast data dissemination in VANETS (ACM Low Wood Bay, Lake District, 2012), p. 53

5. RS Schwartz, RRR Barbosa, N Meratnia, G Heijenk, H Scholten, A directional data dissemination protocol for vehicular environments. Comput. Commun. 34(17), 2057-2071 (2011)

6. O Tonguz, N Wisitpongphan, F Bai, DV-CAST: A distributed vehicular broadcast protocol for vehicular ad hoc networks. IEEE Wireless Commun. 17(2), 47-57 (2010)

7. W Viriyasitavat, F Bai, OK Tonguz, in IEEE Vehicular Networking Conference (VNC). UV-CAST: an urban vehicular broadcast protocol (IEEE Jersey City, 2010), pp. 25-32

8. B Williams, T Camp, in Proceedings of the 3rd ACM international Symposium on Mobile Ad Hoc Networking \& Computing (MobiHoc). Comparison of broadcasting techniques for mobile ad hoc networks (ACM Lausanne, 2002), p. 194

9. H Lim, C Kim, in Proceedings of the 3rd ACM International Workshop on Modeling, Analysis and Simulation of Wireless and Mobile Systems (MSWIM).
Multicast tree construction and flooding in wireless ad hoc networks (ACM Boston, 2000), pp. 61-68

10. W Peng, X Lu, in First Annual Workshop on Mobile and Ad Hoc Networking and Computing (MobiHOC). On the reduction of broadcast redundancy in mobile ad hoc networks (IEEE Boston, 2000), pp. 129-130

11. W Peng, Efficient broadcast in mobile ad hoc networks using connected dominating sets. J. Softw. (1999)

12. M Torrent-Moreno, J Mittag, P Santi, H Hartenstein, Vehicle-to-vehicle communication: fair transmit power control for safety-critical information. IEEE Trans. Vehicular Technol. 58(7), 3684-3703 (2009)

13. S Panichpapiboon, W Pattara-atikom, A review of information dissemination protocols for vehicular ad hoc networks. IEEE Commun. Surv. Tutorials 14(3), 784-798 (2011)

14. H Füß ler, J Widmer, M Käsemann, M Mauve, H Hartenstein, Contention-based forwarding for mobile ad hoc networks. Ad Hoc Netw. 1(4), 351-369 (2003)

15. G Korkmaz, E Ekici, F Özgüner, U Özgüner, in Proceedings of the first ACM Workshop on Vehicular Ad Hoc Networks (VANET). Urban multi-hop broadcast protocol for inter-vehicle communication systems (ACM Philadelphia, 2004), pp. 76-85

16. N Cenerario, T Delot, S llarri, A content-based dissemination protocol for VANETs: exploiting the encounter probability. IEEE Trans. Intell. Transportation Syst. 12(3), 771-782 (2011)

17. J Blum, A Eskandarian, Avoiding timeslot boundary synchronization for multihop message broadcast in vehicular networks. IEEE 69th Vehicular Technol. Conf. (VTC Spring), 1-5. Barcelona, 26-29 April 2009

18. Yt Tseng, Rh Jan, C Chen, Cf Wang, HH Li, in The 7th IEEE International Conference on Mobile Ad-hoc and Sensor Systems (MASS). A vehicle-density-based forwarding scheme for emergency message broadcasts in VANETs (IEEE San Francisco, 2010), pp. 703-708

19. TTielert, D Jiang, Q Chen, L Delgrossi, $H$ Hartenstein, in IEEE Vehicular Networking Conference (VNC). Design methodology and evaluation of rate adaptation based congestion control for Vehicle Safety Communications (IEEE Amsterdam, 2011), pp. 116-123

20. R Meireles, P Steenkiste, J Barros, in IEEE Vehicular Networking Conference (VNC). DAZL: Density-Aware Zone-based packet forwarding in vehicular networks (IEEE Seoul, 2012), pp. 234-241

21. M Koubek, S Rea, D Pesch, Reliable delay constrained multihop broadcasting in VANETs. EURASIP J. Adv. Signal Process. 2010(753256) $1-13$ (2010)

22. A Vahdat, D Becker, Epidemic routing for partially connected ad hoc networks. Tech. rep., Technical Report CS-200006. Duke University (2000)

23. T Spyropoulos, CS Psounis K asd Raghavendra, Efficient routing in intermittently connected mobile networks: the multiple-copy case. IEEE/ACM Trans. Netw. (TON) 16,77-90 (2008)

24. P Hui, J Crowcroft, E Yoneki, in Proceedings of the 9th ACM International Symposium on Mobile Ad Hoc Networking and Computing. Bubble rap: social-based forwarding in delay tolerant networks (ACM Hong Kong, 2008), pp. 241-250

25. FJ Ros, PM Ruiz, I Stojmenovic, in IEEE 69th Vehicular Technology Conference (VTC Spring). Reliable and Efficient Broadcasting in Vehicular Ad Hoc Networks (IEEE Barcelona, 2009), pp. 1-5

26. M Fogue, P Garrido, FJ Martinez, JC Cano, CT Calafate, P Manzoni, Evaluating the impact of a novel message dissemination scheme for vehicular networks using real maps. Transportation Res. Part C: Emerg. Technol. 25, 61-80 (2012)

27. L Hogie, P Bouvry, M Seredynski, F Guinand, in International Conference on Networking, International Conference on Systems and International Conference on Mobile Communications and Learning Technologies (ICNICONSMCL'06). A Bandwidth-Efficient Broadcasting Protocol for Mobile Multi-hop Ad hoc Networks (IEEE Morne, 2006), pp. 71-71

28. P Ruiz, B Dorronsoro, D Khadraoui, P Bouvry, L Tard, in Proceedings of the 2008 High Performance Computing \& Simulation Conference, vol.5, ed. by WW Smari. BODYF - A Parameterless Broadcasting Protocol Over Dynamic Forest (Nicosia, 14-16 April 2008)

29. Vehicular Technology Society, in IEEE 1609.4. IEEE Standard for Wireless Access in Vehicular Environments (WAVE) - Multi-channel Operation IEEE Vehicular Technology Society (IEEE Piscataway, 2010)

30. Vehicular Technology Society, in IEEE 1609.3. IEEE Standard for Wireless Access in Vehicular Environments (WAVE) - Networking Services (IEEE Piscataway, 2010) 
31. Vehicular Technology Society, in IEEE 1609.2. IEEE Standard for Wireless Access in Vehicular Environments - Security Services for Applications and Management Messages (IEEE Piscataway, 2010)

32. M van Eenennaam, W Klein Wolterink, G Karagiannis, G Heijenk, in IEEE Vehicular Networking Conference (VNC). Exploring the solution space of beaconing in VANETs (IEEE Tokyo, 2009), pp. 1-8

33. Department of Defense USA, Global Positioning System Standard. Tech. Rep. Department of Defense USA (2008)

34. A Goldsmith, Wireless Communications. (Cambridge University Press, Cambridge, 2005)

35. C Sommer, D Eckhoff, R German, F Dressler, in Eighth International Conference on Wireless On-Demand Network Systems and Services. A computationally inexpensive empirical model of IEEE $802.11 \mathrm{p} \mathrm{radio}$ shadowing in urban environments (IEEE Bardonecchia, 2011), pp. 84-90

36. P Fuxjäger, A Costantini, D Valerio, P Castiglione, G Zacheo, T Zemen, F Ricciato, in 6th Karlsruhe WSR IEEE 802.11 p transmission using GNURadio (Karlsruhe, 03-04 March 2010), pp. 1-4

37. ETSI Standard, Final draft ETSI ES 202663 V1.1.0 - Intelligent Transport Systems (ITS); European profile standard for the physical and medium access control layer of Intelligent Transport Systems operating in the 5 GHz frequency band. Tech. rep., ETSI, (2009)

38. K Sjöberg, J Karedal, M Moe, ØKristiansen, R Sø råsen, E Uhlemann, F Tufvesson, K Evensen, EG Ström, in 17th World Congress on Intelligent Transport Systems, vol. 2010. Measuring and using the RSSI of IEEE 802.11p (Busan, 25-29 October 2010), pp. 1-9

39. T Rappaport, Wireless Communications: Principles and Practice, vol. 2. (Prentice Hall PTR, Upper Saddle River, 2001)

40. M Behrisch, L Bieker, J Erdmann, D Krajzewicz, in The Third International Conference on Advances in System Simulation (SIMUL). SUMO - Simulation of Urban MObility - an Overview (IARIA Barcelona, 2011), pp. 55-60

doi:10.1186/1687-1499-2013-257

Cite this article as: Schwartz et al:: A scalable data dissemination protocol for both highway and urban vehicular environments. EURASIP Journal on Wireless Communications and Networking 2013 2013:257.

\section{Submit your manuscript to a SpringerOpen ${ }^{\circ}$ journal and benefit from:}

- Convenient online submission

- Rigorous peer review

- Immediate publication on acceptance

- Open access: articles freely available online

- High visibility within the field

- Retaining the copyright to your article 